\title{
The deep-water macroalgal community of the East Florida continental shelf (USA)*
}

\author{
M. Dennis Hanisak \& Stephen M. Blair \\ Marine Botany Department, Harbor Branch Oceanographic Institution; \\ 5600 Old Dixie Highway, Fort Pierce, FL 34946, USA
}

\begin{abstract}
The deep-water macroalgal community of the continental shelf off the east coast of Florida was sampled by lock-out divers from two research submersibles as part of the most detailed year-round study of a macroalgal community extending below routine SCUBA depths. A total of 208 taxa (excluding crustose corallines) were recorded; of these, $42(20.2 \%), 19(9.1 \%)$, and $147(70.7 \%)$ belonged to the Chlorophyta, Phaeophyta, and Rhodophyta, respectively. Taxonomic diversity was maximal during late spring and summer and minimal during late fall and winter. The number of reproductive taxa closely followed the number of taxa present; when reproductive frequency was expressed as a percentage of the species present during each month, two peaks (January and August) were observed. Most perennial species had considerable depth ranges, with the greatest number of taxa observed from 31 to $40 \mathrm{~m}$ in depth. Although most of the taxa present also grow in shallow water (i.e. $<10 \mathrm{~m}$ ), there were some species whose distribution is limited to deeper water. The latter are strongly dominated by rhodophytes. This community has a strong tropical affinity, but over half the taxa occur in warm-temperate areas. Forty-two new records $(20 \%$ of the taxa identified) for Florida were listed; this includes 15 taxa which' previously had been considered distributional disjuncts in this area. The phaeophyte Halopteris filicina is a new addition to the flora of the western Atlantic. Although more studies are needed, it appears that the deep-water flora may be relatively continuous over a large portion of the tropical and subtropical western North Atlantic. The traditional practice of considering Cape Canaveral as a biogeographical boundary may only be appropriate for shallow water. This study demonstrates the need for additional floristic work on macroalgal communities, especially from deep water, before more detailed biogeographical analyses can be performed and suggests that deep-water species could be important in experimental approaches to macroalgal biogeography.
\end{abstract}

\section{INTRODUCTION}

A major breakthrough in the study of deep-water macroalgae has been the development of research submersibles (Earle, 1985). Although phycological studies employing submersibles have been limited, it appears that deep-water algal communities are especially well-developed in the tropical and subtropical western North Atlantic (Littler et al., 1985, 1986; Hanisak \& Blair, in press). These communities include many species that previously have been considered rare or not described at all (Hanisak \& Blair, in press).

Early collections of deep-water macroaigae in the tropical and subtropical western

- Paper presented at the XIV International Botanical Congress (Berlin, 24 July-1 August, 1987), Symposium 6-15, "Biogeography of marine benthic algae". 
North Atlantic were made by naturalists such as Louis Agassiz and Archibald Menzies. Taylor $(1930,1942)$ and Howe \& Taylor (1931) recorded many new species, some of which are still only known from their original collections. In the Gulf of Mexico, Taylor (1928)

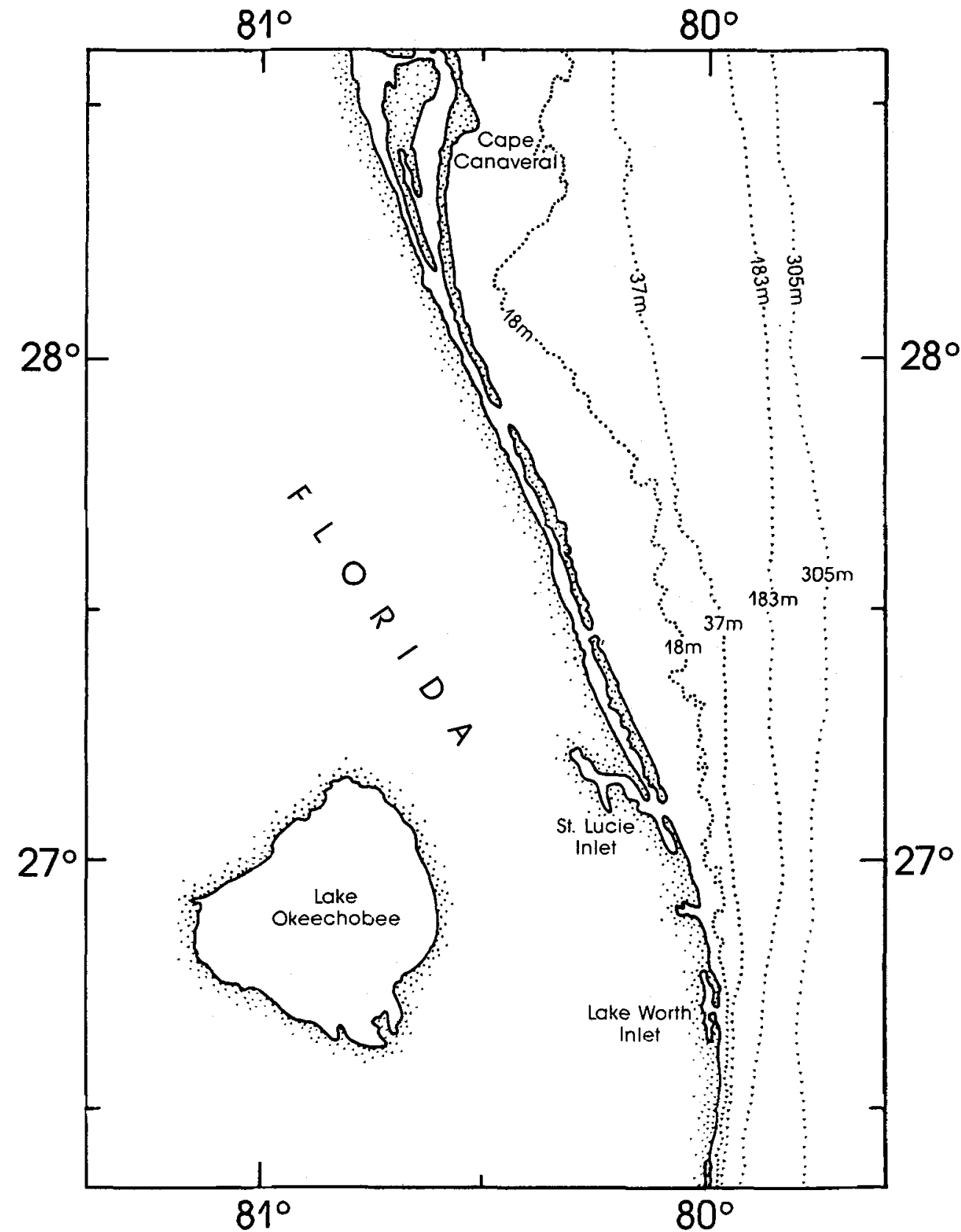

Fig. 1. Map of the study area. Collections were made east of Lake Worth, Jupiter, and St. Lucie Inlets, approximately on the $80^{\circ}$ longitude line. Depths are in meters (redrawn from Avent \& Stanton, 1979) 
collected benthic macroalgae via dredging to depths greater than $100 \mathrm{~m}$. More recent work (Dawes \& van Breedveld, 1969; Cheney \& Dyer, 1974; Eiseman \& Blair, 1982) has added to the available knowledge of the deep-water macroalgae of the Gulf.

The most extensive study of deep-water macroalgae in the western Atlantic has been made along the continental shelf of North Carolina (Schneider, 1974, 1975a, b, 1976, 1984; Schneider \& Reading, in press; Schneider \& Searles, 1973, 1975, 1976; Searles, 1972, 1984a, b; Searles \& Schneider, 1978, 1980) and South Carolina (Wiseman \& Schneider, 1977; Schneider \& Eiseman, 1979; Blair \& Hall, 1981). Yet despite the tremendous activity on the Carolina flora, relatively scant attention has been paid to macroalgae off the extensive eastern coast of Florida, with investigations having concentrated on relatively shallow-water communities (e.g. Phillips, 1961; Juett et al., 1976; Benz et al., 1979; Hall \& Eiseman, 1981).

Given the lack of knowledge of the deep-water macroalgal community of the continental shelf off the coast of Florida (USA), Eiseman (1978) initiated a survey of this community with the research submersibles Johnson-Sea-Link I and Johnson-Sea-Link II in an area from St. Lucie Inlet to the Lake Worth Inlet near Palm Beach (Fig. 1). Eiseman and his colleagues described several new species from these collections (Schneider \& Eiseman, 1979; Eiseman, 1979; Eiseman \& Moe, 1981; Eiseman \& Norris, 1981), but no detailed report of these collections was ever made. During studies of the deep-water macroalgal communities in nearby Bahamian waters (Hanisak \& Blair, in press; Hanisak et al., unpubl.), the value of the earlier Florida collections became apparent. As part of a project to catalogue and verify the holdings of the Harbor Branch Herbarium (HBFH), an opportunity arose to review and reexamine these earlier collections.

This paper presents information on the seasonal and depth distribution, reproductive phenology, and biogeographic affinities of the macroalgal community of the eastern continental shelf of Florida. This study is particularly important for two reasons: (1) it is the most detailed year-round study on a macroalgal community extending below routine SCUBA depths, and (2) the study site is located near Cape Canaveral, an important biogeographic boundary that has been designated as delineating the warm-temperate and tropical floras (Humm, 1969; van den Hoek, 1975, 1984; Searles, 1984b).

\section{MATERIALS AND METHODS}

The deep-water macroalgal community of the east Florida continental shelf was sampled during a series of submersible cruises initiated by Dr. N. J. Eiseman (Eiseman, 1978). The principal collecting sites were located off of Lake Worth Inlet, with less frequent sampling off of St. Lucie and Jupiter Inlets (Fig. 1). Although the substratum at these sites is primarily sand with few algae present, benthic macroalgae do grow on occasional areas of rubble or reefs. These sites were sampled at irregular intervals from July 1974 to June 1983. All months of the year were sampled during these collections with the exception of February.

Seawater temperature was measured during some, but not all collections. Mean monthly water temperatures at depths of $30-50 \mathrm{~m}$ were between 20 and $25^{\circ} \mathrm{C}$ for all months. Temperatures were more variable at greater depths, but usually ranged from 15 to $20^{\circ} \mathrm{C}$ between 60 and $92 \mathrm{~m}$. Significantly lower temperatures $\left(9-12^{\circ} \mathrm{C}\right)$ were occasio- 
nally observed during the summer months at these greater depths when periods of upwelling occurred.

Collections were made by lock-out divers from the research submersibles JohnsonSea-Link I and Johnson-Sea-Link II at depths of ca 24-98 m. Occasional collections at shallower depths were not included in this communication. In total, 171 submersible dives were made on 34 cruises; most of the effort (23 cruises, 143 submersible dives) was concentrated during 1976-1979, when at least 4 cruises a year, with at least one cruise each season, were made. Collection depths were measured by a pressure gauge on the submersible and were accurate to $\pm 0.5 \mathrm{~m}$. The algae were rough sorted aboard ship, preserved in $5 \%$ seawater-formalin, and retumed to the laboratory for identification. Presence of reproductive structures was noted when observed. Following the initial identification of the specimens, voucher specimens were deposited in the Harbor Branch Herbarium (HBFH).

During 1984, we initiated the first efforts at cataloguing the contents of $\mathrm{HBFH}_{\text {; }}$ a large portion of its holdings was from the collections initiated by Eiseman. Despite the obvious importance of these collections, only a few species descriptions and range extensions have been reported (Eiseman, 1979; Schneider \& Eiseman, 1979; Eiseman \& $\mathrm{Moe}_{3}$ 1981; Eiseman \& Norris, 1981). However, before even a species list of these collections could be generated, it was necessary to verify and update identifications previously made. In this process, ca 4000 records were examined. This effort was expedited significantly by Wynne's checklist (1986) that synthesized the recent taxonomic changes made in the tropical and subtropical western Atlantic.

Our systematic list within macroalgal divisions follows that of Wynne (1986); crustose corallines are not included. Monthly occurrence and reproductive phenology as well as the mean depth and range of depths for each taxon'were tabulated. Because of the nearness of the site to the important "biogeographical boundary" at Cape Canaveral (located ca $150-190 \mathrm{~km}$ north of the stations in this study), we were interested in determining the "biogeographic affinity" of the individual species in the macroalgal community. For this biogeographical information, we relied primarily on species lists and distribution records previously prepared by Kapraun $(1980,1984)$, Searles \& Schneider (1978), South \& Tittley (1986), and Taylor (1960). Additional information on these biogeographic affinities, as well as previous records for Florida, was obtained from numerous references cited by Wynne (1986).

\section{RESULTS}

A total of 208 taxa were recorded during this study (Table 1). Of these $42(20.2 \%), 19$ $(9.1 \%)$, and $147(70.7 \%)$ belonged to the Chlorophyta, Phaeophyta, and Rhodophyta, respectively (Table 2). A significant portion of these species was found year-round; less frequently occurring species were usually collected during the summer (Table 3). The number of taxa (Table 2) was highest during late spring and summer (May-July) and lowest during late fall and winter (November-January). Although not quantified, macroalgal abundance was visually correlated with macroalgal diversity and had the same seasonal pattern.

Many species of the east Florida continental shelf were reproductive throughout the year, with the number of reproductive taxa highest in the summer (June to August) near 
Table 1. Systematic list (excluding crustose corallines) identified during the study

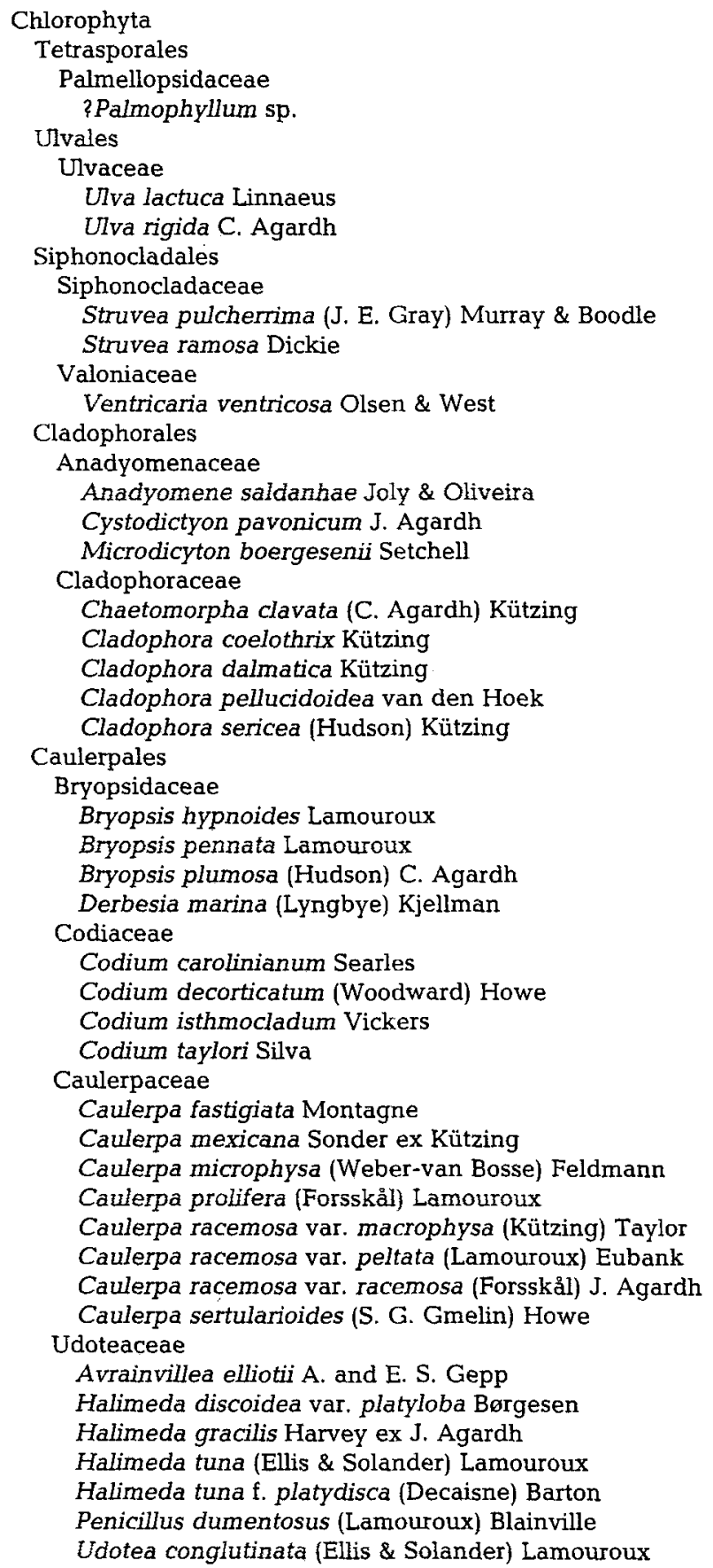


Table 1 (Continued)

Udotea cyathiformis Decaisne

Udotea flabellum (Ellis \& Solander) Lámouroux

Udotea spinulosa Howe

Pseudocodium floridanum Dawes \& Mathieson

Dasycladales

Dasycladaceae

Neomeris annulata Dickie

Phaeophyta

Sporochnales

Sporochnaceae

Nereia tropica (W. Taylor) W. Taylor

Sporochnus bolleanus Montagne

Sporochnus pedunculatus (Hudson) C. Agardh

Scytosiphonales

Scytosiphonaceae

Colpomenia sinuosa (Endlicher) Derbes \& Solier in Castagne

Syringodermatales

Syringodermataceae

Syringoderma floridana Henry

Sphacelariales

Sphacelariaceae

Halopteris filicina (Grateloupe) Kützing

Dictyotales

Dictyotaceae

Dictyopteris delicatula Lamouroux

Dictyopteris hoytii W. Taylor

Dictyopteris plagiogramma (Montagne) Vickers

Dictyota bartayresii Lamouroux

Dictyota cervicornis Kützing

Dictyota dichotoma (Hudson) Lamouroux

Dictyota divaricata Lamouroux

Lobophora variegata (Lamouroux) Womersley

Spatoglossum schroederi (C. Agardh) Kützing

Fucales

Stypopodium zonale (Lamouroux) Papenfuss

Sargassaceae

Sargassum cymosum C. Agardh

Sargassum hystrix J. Agardh

Sargassum hystrix var. buxifolium Chauvin in J. Agardh

Rhodophyta

Compsopogonales

Erythropeltidaceae

Erythrotrichia carnea (Dillwyn) J. Agardh

Nemaliales

Acrochaetiaceae

Audouinella daviesii (Dillwyn) Woelkerling

Galaxauraceae

Galaxaura marginata (Ellis \& Solander) Lamouroux

Galaxaura oblongata (Ellis \& Solander) Lamouroux

Galaxaura obtusata (Ellis \& Solander) Lamouroux

Scinaia complanata (Collins) Cotton

Scinaia complanata var. intermedia Børgesen

Scinaia incrassata Eiseman 
Table 1 (Continued)

Gelidiales

Gelidiaceae

Gelidium americanum (W. Taylor) Santelices

Pterocladia capillacea (S. G. Gmelin) Bornet \& Thuret

Bonnemaisoniales

Bonnemaisoniaceae

Asparagopsis taxiformis (Delile) Trevisan (Falkenbergia stage)

Corallinales (excluding crustose forms)

Corallinaceae

Amphiroa rigida var. antillana Børgesen

Amphiroa tribulus (Ellis \& Solander) Lamouroux

Jania adhaerens Lamouroux

Gigartinales

Nemastomataceae

Nemastoma gelatinosum Howe

Platoma cyclocolpa (Montagne) Schmitz

Predaea feldmannii G. De Toni

Predaea masonii (Setchell \& Gardiner) G. De Toni

Titanophora incrustans (J. Agardh) Børgesen

Hypneaceae

Hypnea cervicornis J. Agardh

Hypnea spinella (C. Agardh) Kützing

Hypnea volubilis Searles

Wurdemanniaceae

Wurdemannia miniata (Duby) J. Feldmann \& Hamel

Solieriaceae

Eucheuma isiforme (C. Agardh) J. Agardh

Meristotheca floridana Kylin

Sarcodiotheca caribaea W. Taylor

Solieria filiformis (Kützing) Gabrielson

Gracilariaceae

Gelidiopsis planicaulis (W. Taylor) W. Taylor

Gracilaria ?cuneata Areschoug

Gracilaria curtissiae J. Agardh

Gracilaria mammilaris (Montagne) Howe

Gracilaria occidentalis (Børgesen) Bodard

Cryptonemiales

Peyssonneliaceae

Peyssonnelia inamoena Pilger

Dumontiaceae

Acrosymphyton caribaeum (J. Agardh) Sjostedt

Dudresnaya bermudensis Setchell

Dudresnaya crassa Howe

Dudresnaya patula Eiseman \& J. Norris

Halymeniaceae

Cryptonemia crenulata (J. Agardh) J. Agardh

Cryptonemia luxurians (C. Agardh) J. Agardh

Cryptonemia sp.

Halymenia agardhii De Toni

Halymenia bermudensis Collins \& Hervey

Halymenia floresia (Clemente) C. Agardh

Halymenia floridana J. Agardh

Halymenia gelinaria Collins \& Howe 
Table 1 (Continued)

Halymenia hancockii W. Taylor

Halymenia integra Howe \& W. Taylor

Halymenia rosacea Howe \& W. Taylor

Halymenia vinacea Howe \& W. Taylor

Kallymeniaceae

Callophyllis sp.

Cirrulicarpus carolinensis Hansen

Kallymenia sp.

Kallymenia westii Ganesan

Rhodymeniales

Champiaceae

Champia parvula (C. Agardh) Harvey

Lomentaria baileyana (Harvey) Farlow

Rhodymeniaceae

Agardhinula browneae (J. Agardh) De Toni

Botryocladia occidentalis (Børgesen) Kylin

Botryocladia pyriformis (Børgesen) Kylin

Chrysymenia agardhii Harvey

Chrysymenia enteromorpha Harvey

Chrysymenia halymenioides Harvey

Chrysymenia planifrons (Melvill) J. Agardh

Coelarthrum albertisii (Piccone) Børgesen

Gloioderma atlanticum Searles

Gloioderma blomquistii Searles in Schneider \& Searles

Gloioderma rubisporum Searles

Halichrysis peltata (W. Taylor) P. Huvé \& H. Huvé

Leptofauchea rhodymenioides W. Taylor

Maripelta atlantica Eiseman \& Moe

Rhodymenia divaricata Dawson

Rhodymenia pseudopalmata (Lamouroux) Silva

Ceramiales

Ceramiaceae

Anotrichium barbatum (J. E. Smith) Nägeli

Anotrichium tenue (C. Agardh) Nägeli

Antithamnion antillanum Børgesen

Antithamnion cherminieri (Nasr)

Antithamnionella atlantica (Oliviera) Schneider

Antithamnionella breviramosa (Dawson) Wollaston in Womersley \& Bailey

Antithamnionella flagellata (Børgesen) Abbott

Antithamnionella ?latiaxis Abbott

Antithamnionella sp.

Balliella psendocorticata (Dawson) D. Young

Callithamniella tingitana (Schousboe ex Bornet) Feldman-Mazoyer

Callithamnion cordatum Børgesen

Callithamnion halliae Collins

Callithamnion uruguayense W. Taylor

Centroceras clavulatum (C. Agardh in Kunth) Montagne in Durieu de Maisonneuve

Ceramium comptum Bargesen

Ceramium fastigiatum f. flaccida $\mathrm{H}$. Petersen

Ceramium flaccidum (Kützing) Ardissone

Ceramium leptozonum Howe

Ceramium sp.

Compsothamnion thuyoides (J. E. Smith) Schmitz 
Table 1 (Continued)

\begin{tabular}{|c|}
\hline 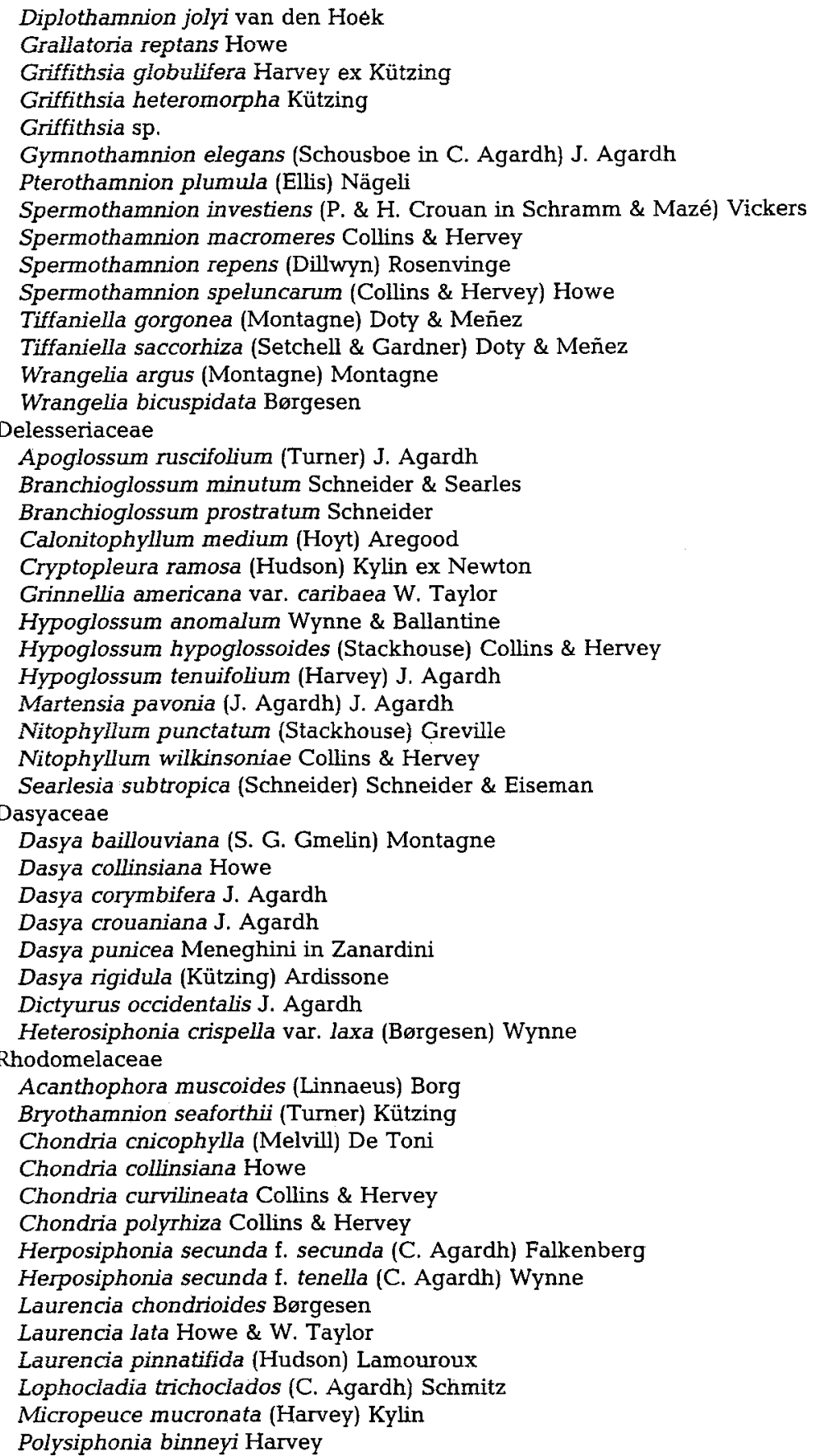 \\
\hline
\end{tabular}


Table 1 (Continued)

\author{
Polysiphonia ferulacea Suhr ex J. Agardh \\ Polysiphonia havanensis Montagne \\ Polysiphonia sphaerocarpa Børgesen \\ Waldoia antillana W. Taylor \\ Wrightiella blodgettii (Harvey) Schmitz \\ Wrightiella tumanowiczii (Gatty) Schmitz
}

the period of maximal taxonomic diversity (Table 2). However, when reproductive frequency was expressed as a percentage of reproductive species to the number of species present in each month, two peaks (January and August) were apparent (Table 2).

In terms of depth distribution, the number of taxa recorded was highest at depths of 31-40 $\mathrm{m}$ (Table 4) and decreased as depth increased; this decline was more gradual for rhodophytes than for chlorophytes and phaeophytes. Most perennial species had considerable depth ranges (Table 5). Only two chlorophytes had a mean depth greater than $50 \mathrm{~m}$; one of these was Bryopsis hypnoides which was collected only once during this study, and the other was ?Palmophyllum sp. which was collected frequently and was the deepest occurring chlorophyte, being found to depths of $92 \mathrm{~m}$. Similarly, although six phaeophytes had mean depths in excess of $50 \mathrm{~m}$, five of these (Dictyopteris hoytii, Nereia tropica, Sporochnus bolleanus, $S$. pedunculatus, and Syringoderma floridana) seldom occurred; only one brown alga, Halopteris filicina, was collected regularly at depths greater than $50 \mathrm{~m}$. Like ?Palmophyllum sp., $H$. filicina was collected at depths greater than $90 \mathrm{~m}$. As depth increased, the relative proportion of red algae present in the community increased; 25 species of rhodophytes had a mean depth greater than $50 \mathrm{~m}$. Of these, a perennial assemblage of deep-water rhodophytes was apparent and consisted of Apoglossum ruscifolium, Compsothamnion thuyoides, Halymenia gelinaria, H. hancockii, Kallymenia sp., Maripelta atlantica, Nitophyllum punctatum, Peyssonnelia inamoena, and Searlesia subtropica. Three species (A. ruscifolium, $P$. inamoena, and $S$. subtropica) were found at the greatest depth sampled $(98 \mathrm{~m})$.

Examination of previously published distribution records established the biogeographical affinities of the taxa present on the east Florida continental shelf (Table 5). Approximately half of the taxa were restricted to one biogeographical zone, but the rest occurred in two or three biogeographical zones along the east coast of the Americas (Table 6). The flora of the east Florida continental shelf has an obvious tropical affinity (Tables 5,6$) ; 87 \%$ of the taxa in this study were previously collected in tropical waters. This tropical affinity was consistent for the three divisions (85.0-92.9\%). A strong warmtemperate element $(52.4 \%$ of the total flora have been reported from warm-temperate waters north of Florida; $42.1-55.8 \%$ range for the three divisions) is also present, but less than $10 \%$ of the taxa have been reported from cold-temperate waters.

Five taxa are currently considered to occur only in transitional areas between the warm temperate and tropical zones (Table 6); these are Halopteris filicina (new record for the western Atlantic); Dudresnaya patula, Scinaia incrassata, Syringoderma floridana (only reported for the east Florida continental shelf); and Maripelta atlantica (limited to transitional areas in Florida, both east and west coasts).

Forty-two new records for Florida were found during this study (Table 5, 7); this is 


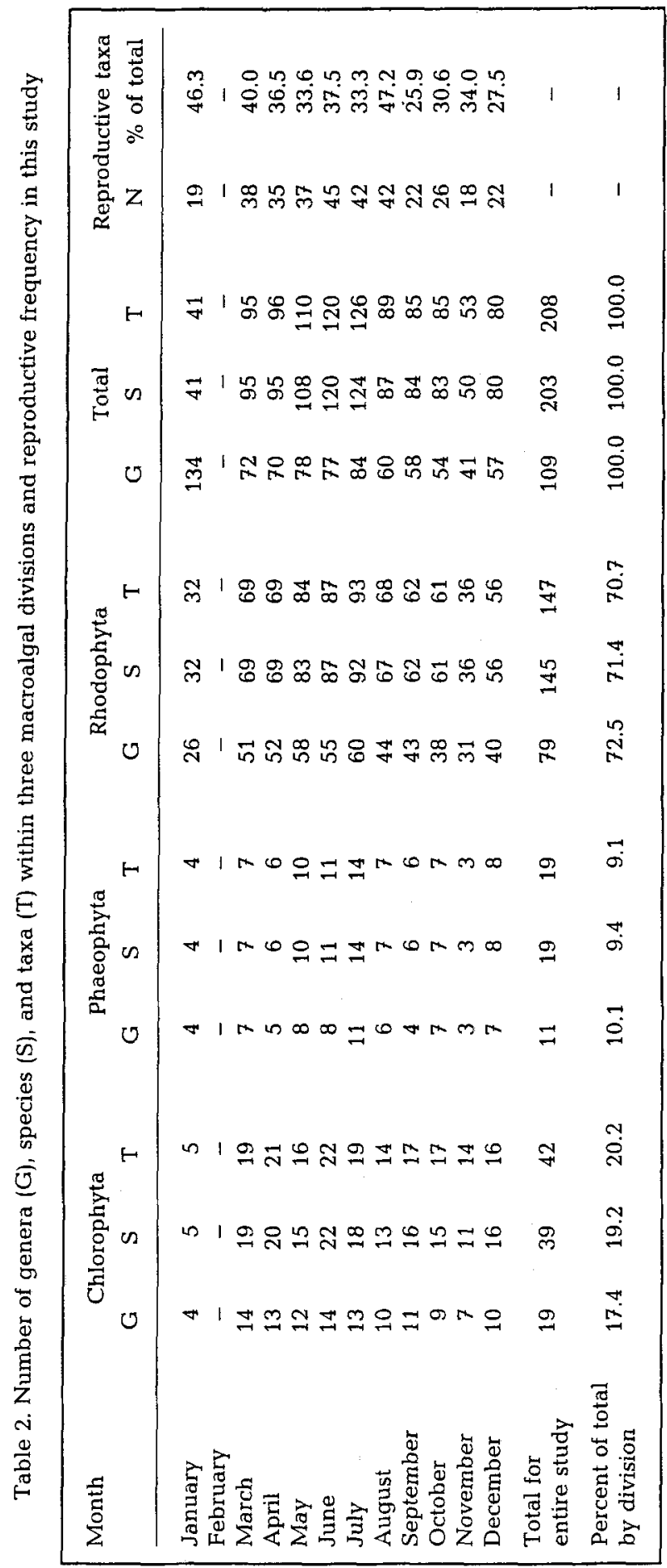




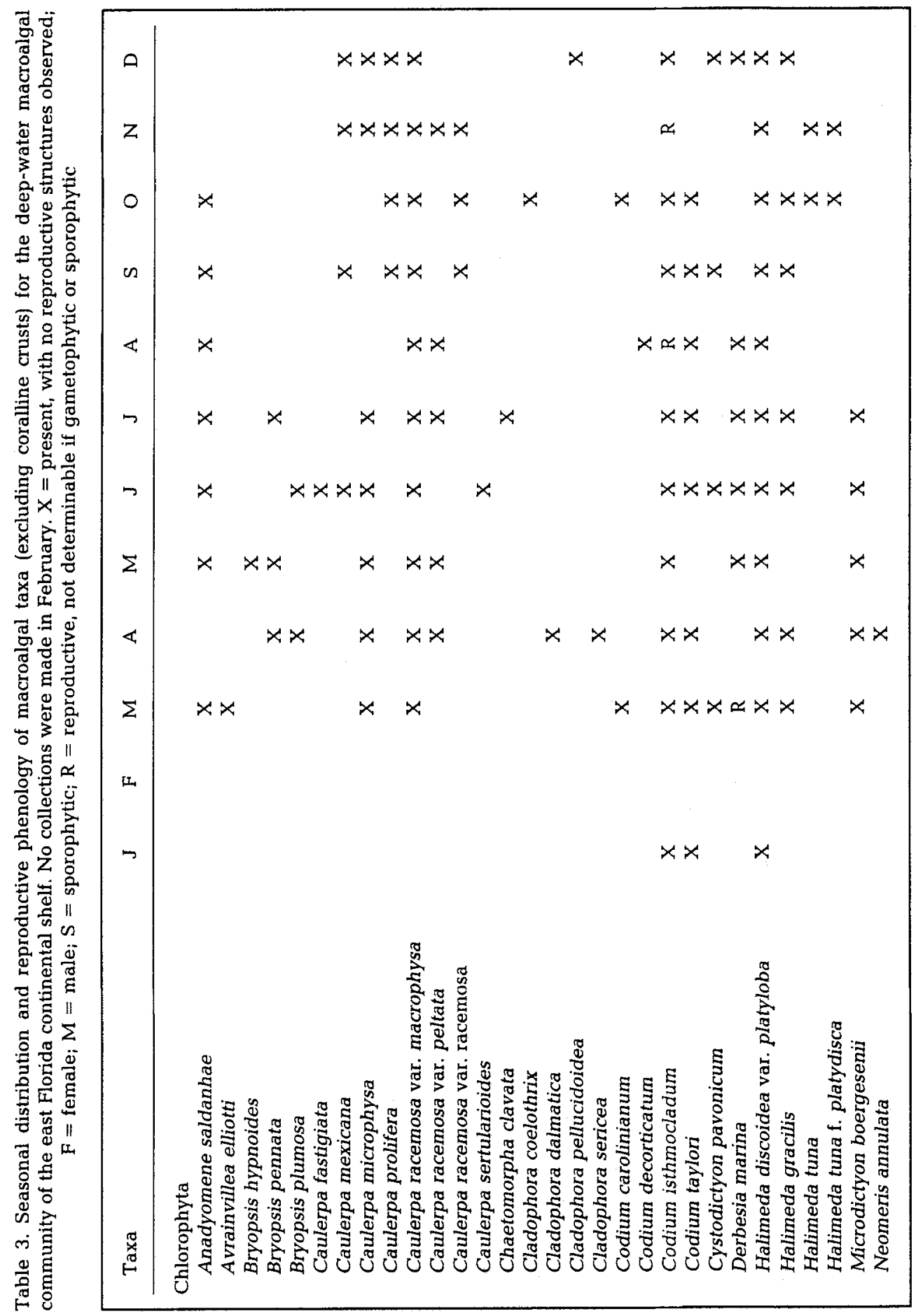




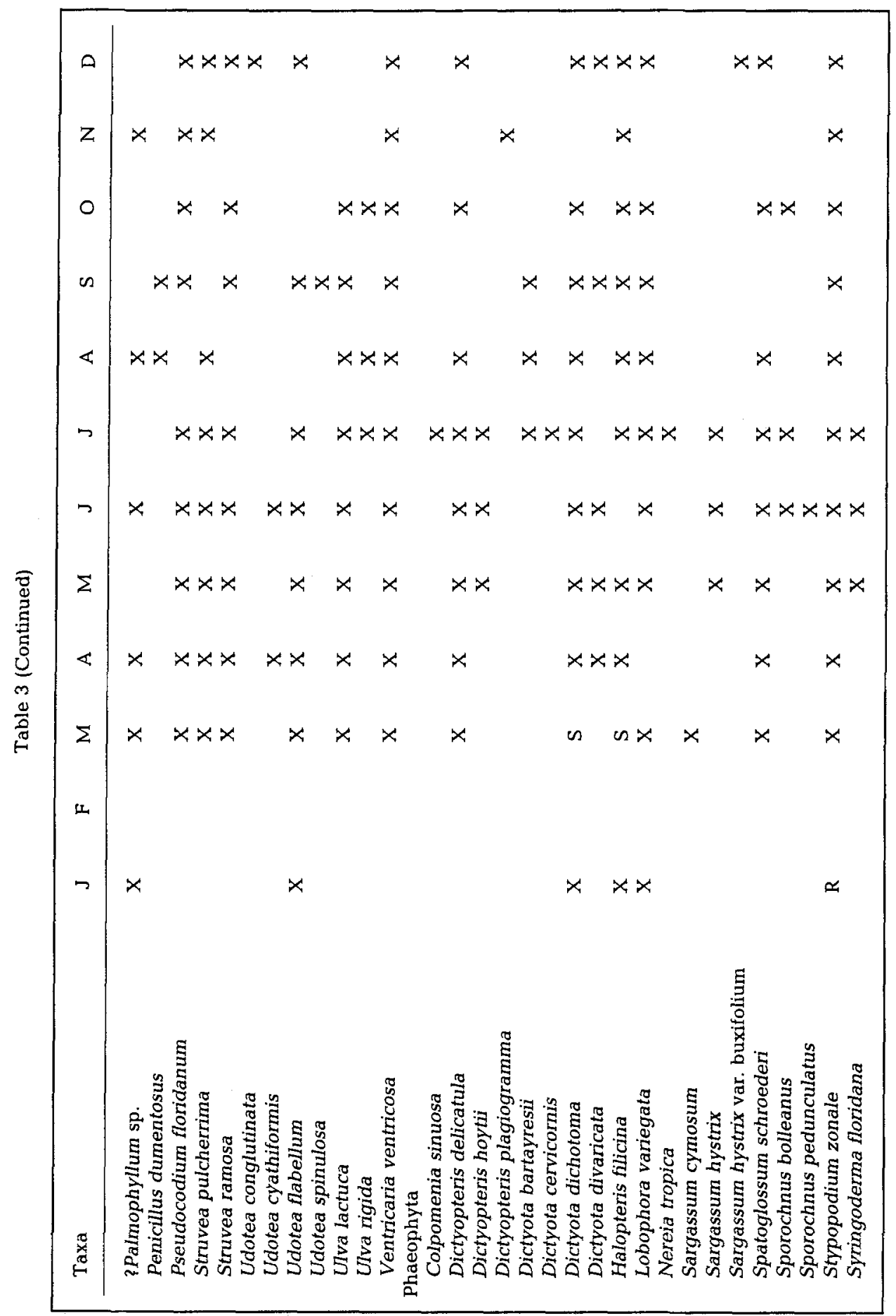




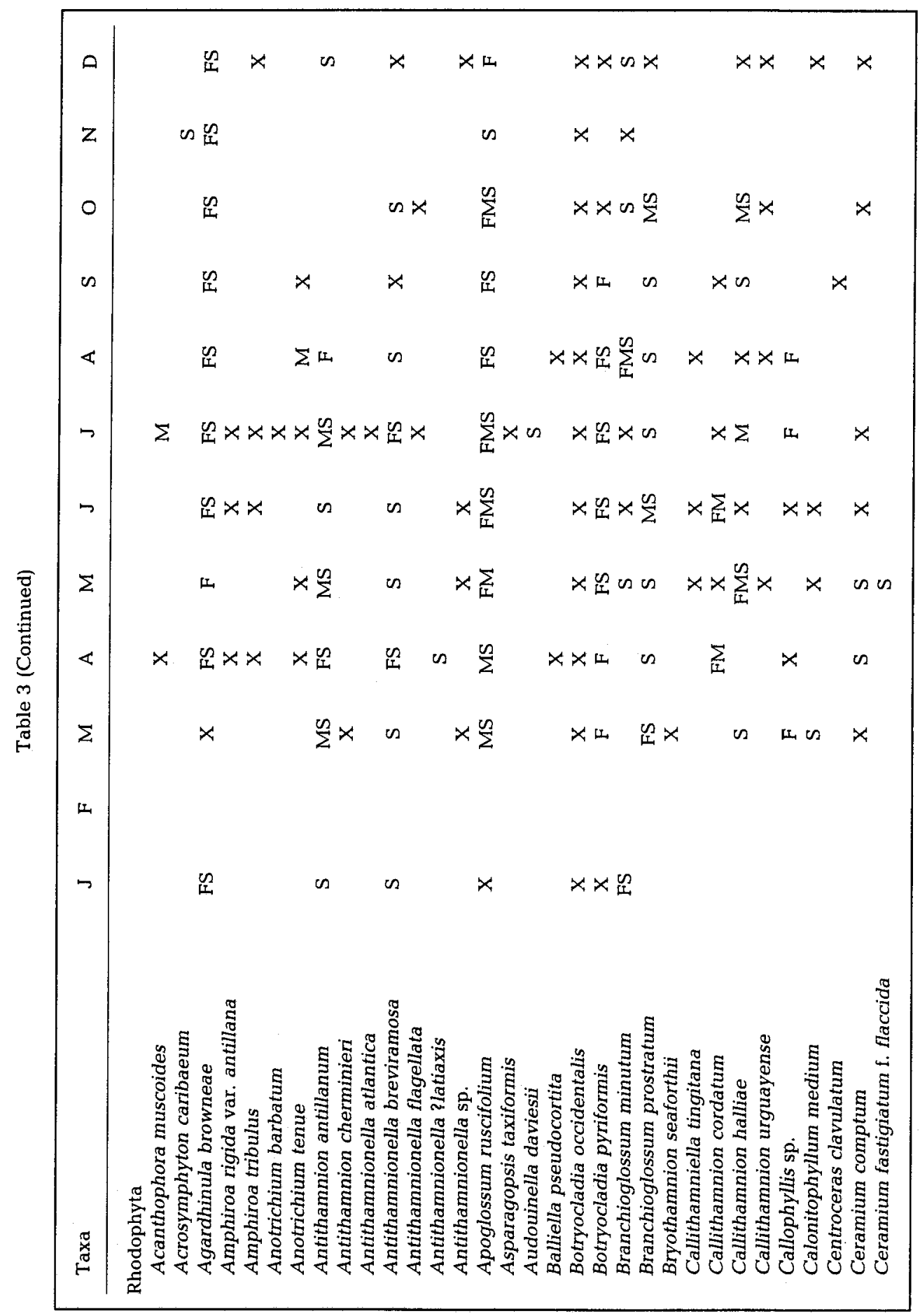




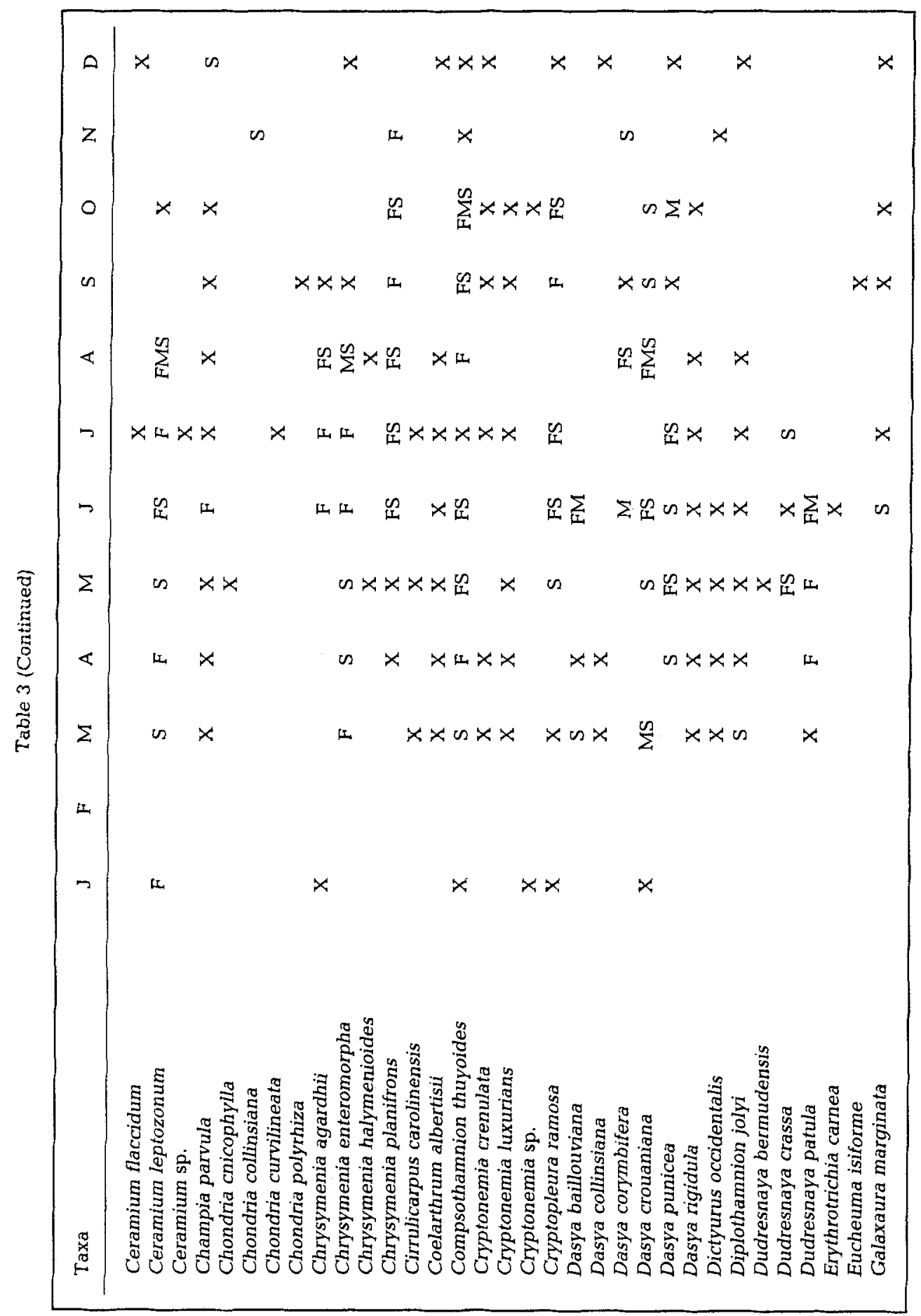




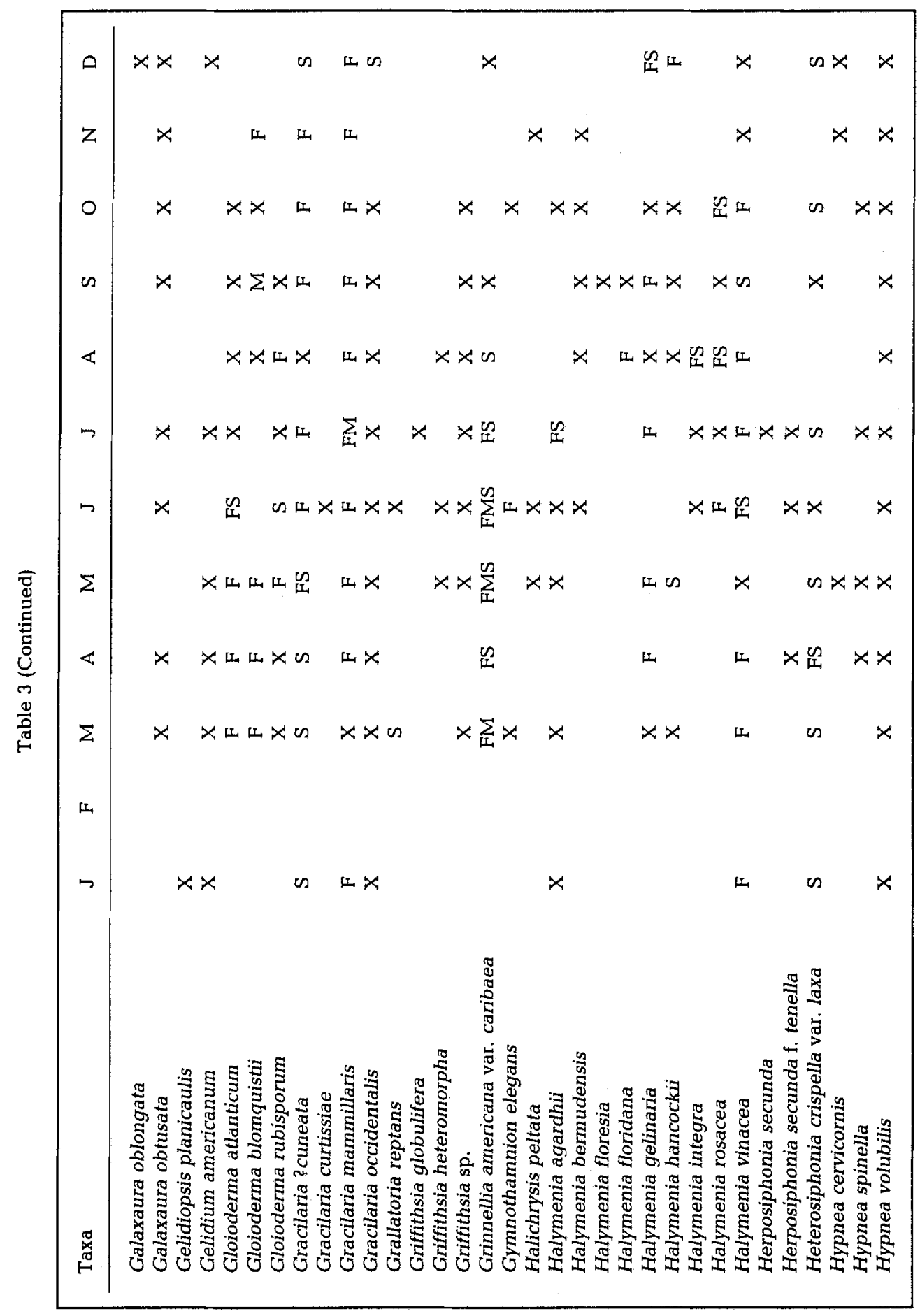




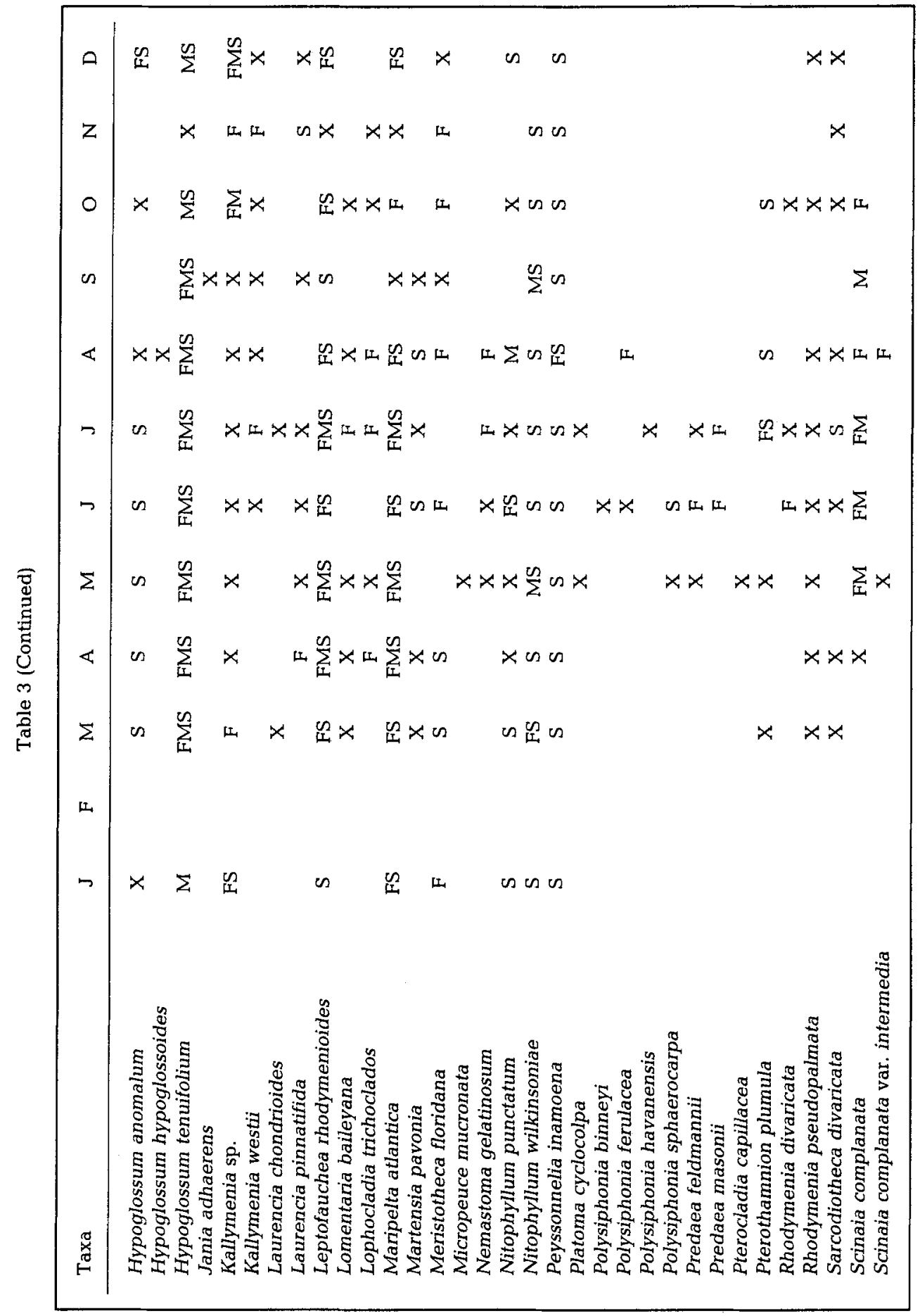




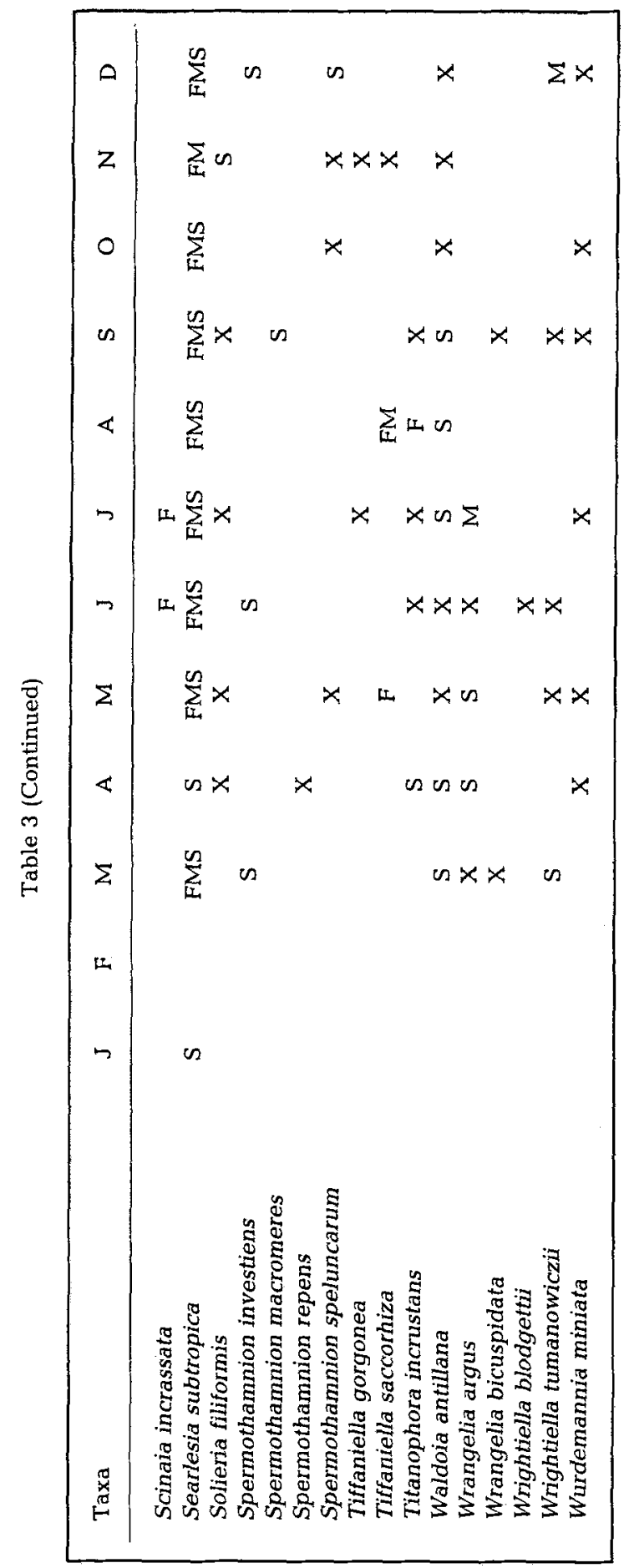


Table 4. Number of macroalgal taxa (excluding crustose corallines) as a function of depth on the east Florida continental shelf

\begin{tabular}{|c|c|c|c|c|c|c|c|}
\hline \multirow{2}{*}{$\begin{array}{l}\text { Depth range } \\
\text { (m) }\end{array}$} & \multicolumn{2}{|c|}{ Chlorophyta } & \multicolumn{2}{|c|}{ Phaeophyta } & \multicolumn{2}{|c|}{ Rhodophyta } & \multirow{2}{*}{$\begin{array}{c}\text { Total } \\
\mathrm{n}\end{array}$} \\
\hline & $\mathbf{n}$ & $\%$ of total & $\mathbf{n}$ & $\%$ of total & $\mathbf{n}$ & $\%$ of total & \\
\hline$\leq \mathbf{3 0}$ & 25 & 24.3 & 6 & 5.8 & 74 & 70.5 & 105 \\
\hline $31-40$ & 32 & 20.2 & 12 & 7.6 & 114 & 72.2 & 158 \\
\hline $41-50$ & 25 & 17.9 & 12 & 8.6 & 104 & 73.8 & 141 \\
\hline $51-60$ & 14 & $13: 1$ & 14 & 13.1 & 80 & 74.1 & 108 \\
\hline $61-70$ & 1 & 2.1 & 4 & 8.3 & 44 & 89.8 & 49 \\
\hline $71-80$ & 1 & 2.4 & 4 & 9.5 & 37 & 88.1 & 42 \\
\hline $81-90$ & 1 & 3.6 & 2 & 7.1 & 25 & 89.3 & 28 \\
\hline $91-100$ & 1 & 5.6 & 1 & 5.6 & 16 & 88.9 & 18 \\
\hline
\end{tabular}

$20 \%$ of the flora of the east Florida continental shelf. In addition, Antithamnionella ?latiaxis would be a new record for Florida if its tentative identification is verified. Fifteen of these new records were previously considered to be distribution disjuncts between the well-studied North Carolina flora and more tropical areas to the south. Fourteen records were considered to be northern extensions, and eight records were southern extensions. Halopteris filicina, a European species, is a new addition to the flora of the western Atlantic. The three additional records considered to be western extensions were previously recorded for the Bahamas at the same approximate latitude as that of the sites of our study. In addition, we have confirmed the occurrence of Agardhinula browneae in Florida which Searles (1984b) had considered uncertain because previous specimens were drift material.

Two taxonomical notes should be made. First, on re-examination of the records attributed by Eiseman (1979) to Petroglossum undulatum they have been reassigned to Gracilaria ?cuneata. Thus, we cannot confirm the presence of Petroglossum in Florida. Second, ?Palmophyllum sp. appears to be an undescribed species that we have previously observed in the Bahamas and the Gulf of Mexico (Hanisak, unpublished). It is the same taxon as the undescribed "palmelloid green" of Littler et al. $(1985,1986)$.

\section{DISCUSSION}

The study of deep-water macroalgal communities has been limited by the ability of phycologists to make direct, in situ observations in the sea. Until recently, knowledge of these plants has been limited, primarily restricted to information obtained from dredging records or from collections of rare and unusual algae occasionally cast ashore following severe storms. Various dredging techniques were important in early attempts at assessing the composition of deep-water macroalgal communities (e.g. David et al., 1904; Taylor, 1928; Humm, 1956; Adey \& MacIntyre, 1973; De Wreede \& Jones, 1973; Doty et al., 1974). However, such limited methods provide only a fragmented, and poorly quantified, glimpse of deep-water communities.

With the introduction of SCUBA in the 1950's, phycologists were able to extend their direct observations of macroalgal communities from the intertidal zone to the shallow subtidal (i.e. to depths of ca $30 \mathrm{~m}$ ) and maximally to a depth of ca $65 \mathrm{~m}$ (e.g. Gilmartin, 
Table 5. Mean depth, depth range, and biogeographical affinities of the taxa identified during this study

\begin{tabular}{|c|c|c|c|c|c|}
\hline \multirow[t]{2}{*}{ Species } & \multicolumn{2}{|c|}{ Depth (m) } & \multicolumn{3}{|c|}{ Biogeographical affinities } \\
\hline & Mean & Range & $\begin{array}{c}\text { Cold } \\
\text { temperate }\end{array}$ & $\begin{array}{c}\text { Warm } \\
\text { temperate }\end{array}$ & Tropical \\
\hline \multicolumn{6}{|l|}{ Chlorophyta } \\
\hline Anadyomene saldanhae & 39 & $29-47$ & - & - & $x$ \\
\hline Avrainvillea elliotti & 28 & 28 & - & - & $x$ \\
\hline Bryopsis hypnoides & 54 & 54 & $\mathrm{X}$ & $\mathrm{x}$ & $\mathrm{x}$ \\
\hline Bryopsis pennata & 43 & $34-56$ & - & $\mathrm{X}$ & $\mathrm{X}$ \\
\hline Bryopsis plumosa & 33 & 33 & $\mathrm{X}$ & $\mathrm{X}$ & $X$ \\
\hline Caulerpa fastigiata & 27 & 27 & - & - & $\mathrm{X}$ \\
\hline Caulerpa mexicana & 36 & $24-45$ & - & - & $\mathrm{X}$ \\
\hline Caulerpa microphysa & 33 & $24-56$ & - & - & $\mathrm{X}$ \\
\hline Caulerpa prolifera & 39 & $24-58$ & - & $\mathrm{X}$ & $\mathrm{x}$ \\
\hline $\begin{array}{c}\text { Caulerpa racemosa } \\
\text { var. macrophysa }\end{array}$ & 35 & $24-50$ & - & - & $\mathrm{X}$ \\
\hline $\begin{array}{l}\text { Caulerpa racemosa } \\
\text { var. peltata }\end{array}$ & 40 & $33-44$ & - & $\mathrm{X}$ & $\mathrm{X}$ \\
\hline $\begin{array}{l}\text { Caulerpa racemosa } \\
\text { var. racemosa }\end{array}$ & 35 & $32-39$ & - & - & $\mathrm{X}$ \\
\hline Caulerpa sertularioides & 42 & $39-45$ & - & - & $\mathrm{X}$ \\
\hline Chaetomorpha clavata & 34 & 34 & - & - & $\mathrm{x}$ \\
\hline Cladophora coelothrix & 39 & 39 & - & - & $\mathrm{X}$ \\
\hline Cladophora dalmatica & 25 & 25 & $\mathrm{X}$ & $\mathrm{x}$ & $\mathrm{X}$ \\
\hline Cladophora pellucidoidea & 34 & 34 & - & $\mathrm{X}$ & $\mathrm{x}$ \\
\hline "Cladophora sericea & 49 & 49 & $\mathrm{x}$ & $\mathrm{X}$ & - \\
\hline Codium carolinianum & 34 & 34 & - & $\mathrm{X}$ & - \\
\hline Codium decorticatum & 42 & 42 & - & $\mathrm{x}$ & $\mathrm{X}$ \\
\hline Codium isthmocladum & 40 & $24-58$ & - & $\mathrm{x}$ & $\mathrm{X}$ \\
\hline Codium taylori & 33 & $24-50$ & - & - & $\mathrm{x}$ \\
\hline Cystodictyon pavonicum & 38 & $38-39$ & - & - & $x$ \\
\hline - Derbesia marina & 45 & $28-58$ & $\mathrm{X}$ & $\mathrm{X}$ & - \\
\hline $\begin{array}{l}\text { Halimeda discoidea var. } \\
\text { platyloba }\end{array}$ & 34 & $24-46$ & - & - & $\mathrm{x}$ \\
\hline - Halimeda gracilis & 37 & $26-48$ & - & - & $\mathrm{X}$ \\
\hline Halimeda tuna & 36 & $34-39$ & - & - & $\mathrm{X}$ \\
\hline Halimeda tuna f. platydisca & 39 & $34-43$ & - & - & $\mathrm{X}$ \\
\hline Microdictyon boergesenii & 39 & $25-56$ & - & $\mathrm{X}$ & $\mathrm{X}$ \\
\hline Neomeris annulata & 25 & 25 & - & - & $\mathrm{X}$ \\
\hline *?Palmophyllum sp. & 60 & $27-92$ & - & - & $\mathrm{X}$ \\
\hline Penicillus dumentosus & 28 & $27-28$ & - & - & $x$ \\
\hline Pseudocodium floridanum & 43 & $24-59$ & - & - & $\mathrm{X}$ \\
\hline Struvea pulcherrima & 45 & $24-58$ & - & $\mathrm{X}$ & $\mathrm{X}$ \\
\hline "Struvea ramosa & 49 & $28-59$ & - & $\mathrm{X}$ & $x$ \\
\hline Udotea conglutinata & 39 & 39 & - & $\mathrm{x}$ & $\mathrm{x}$ \\
\hline Udotea cyathiformis & 34 & $25-42$ & - & $\mathrm{X}$ & $\mathrm{X}$ \\
\hline Udotea flabellum & 33 & $26-39$ & - & $\mathrm{X}$ & $\mathrm{X}$ \\
\hline Udotea spinulosa & 27 & 27 & - & - & $x$ \\
\hline Ulva lactuca & 45 & $28-58$ & $\mathrm{X}$ & - & $\mathrm{X}$ \\
\hline Ulva rigida & 50 & $45-58$ & $\mathrm{X}$ & $\mathrm{X}$ & $\mathrm{X}$ \\
\hline Ventricaria ventricosa & 43 & $27-59$ & - & - & $\mathrm{X}$ \\
\hline
\end{tabular}


Table 5 (Continued)

\begin{tabular}{|c|c|c|c|c|c|}
\hline \multirow[t]{2}{*}{ Species } & \multicolumn{2}{|c|}{ Depth (m) } & \multicolumn{3}{|c|}{ Biogeographical affinities } \\
\hline & Mean & Range & $\begin{array}{l}\text { Cold } \\
\text { temperate }\end{array}$ & $\begin{array}{l}\text { Warm } \\
\text { temperate }\end{array}$ & Tropical \\
\hline \multicolumn{6}{|l|}{ Phaeophyta } \\
\hline Colpomenia sinuosa & 48 & $34-71$ & - & $\mathrm{X}$ & $\mathrm{X}$ \\
\hline Dictyopteris delicatula & 41 & $25-59$ & - & $\mathrm{x}$ & $\mathrm{X}$ \\
\hline "Dictyopteris hoytii & 58 & $56-59$ & - & $\mathrm{X}$ & $\mathrm{x}$ \\
\hline Dictyopteris plagiogramma & 24 & 24 & - & - & $\mathrm{x}$ \\
\hline Dictyota bartayresï & 45 & $34-54$ & - & - & $\mathrm{X}$ \\
\hline Dictyota cervicomis & 40 & 40 & - & $\mathrm{X}$ & $\mathrm{X}$ \\
\hline Dictyota dichotoma & 40 & $25-59$ & $\mathrm{X}$ & $\mathrm{x}$ & $\mathrm{x}$ \\
\hline Dictyota divaricata & 36 & $27-58$ & - & - & $\mathrm{X}$ \\
\hline •"Halopteris filicina & 86 & $46-98$ & - & - & - \\
\hline Lobophora variegata & 39 & $29-58$ & - & $\mathrm{x}$ & $\mathrm{x}$ \\
\hline Nereia tropica & 59 & 59 & - & - & $\mathrm{X}$ \\
\hline Sargassum cymosum & 40 & 40 & - & - & $\mathrm{X}$ \\
\hline Sargassum hystrix & 43 & $40-45$ & - & - & $\mathrm{X}$ \\
\hline $\begin{array}{l}\text { Sargassum hystrix var. } \\
\text { buxifolium }\end{array}$ & 38 & 38 & - & - & $\mathrm{x}$ \\
\hline Spatoglossum schroederi & 48 & $38-58$ & - & $\mathrm{x}$ & $\mathrm{X}$ \\
\hline Sporochnus bolleanus & 54 & $45-59$ & _- & - & $\mathrm{x}$ \\
\hline Sporochnus pedunculatus & 62 & $45-80$ & - & $\mathrm{X}$ & $\mathrm{X}$ \\
\hline Stypopodium zonale & 35 & $25-54$ & - & - & $\mathrm{x}$ \\
\hline Syringoderma floridana & 73 & $56-90$ & - & - & - \\
\hline \multicolumn{6}{|l|}{ Rhodophyta } \\
\hline Acanthophora muscoides & 41 & $25-57$ & - & - & $\mathrm{x}$ \\
\hline Acrosymphyton caribaeum & 43 & 43 & - & - & $\mathrm{X}$ \\
\hline Agardhinula browneae & 50 & $34-90$ & - & $\mathrm{X}$ & $\mathrm{X}$ \\
\hline $\begin{array}{l}\text { Amphiroa rigida var. } \\
\text { antillana }\end{array}$ & 27 & $26-27$ & - & - & $\mathrm{X}$ \\
\hline Amphiroa tribulus & 31 & $26-36$ & - & - & $\mathrm{x}$ \\
\hline "Anotrichium barbatum & 59 & 59 & - & $\mathrm{x}$ & $\mathrm{X}$ \\
\hline Anotrichium tenue & 40 & $36-44$ & $\mathrm{x}$ & $\mathrm{x}$ & $\mathrm{X}$ \\
\hline "Antithamnion antillanum & 35 & $25-54$ & - & - & $\mathrm{x}$ \\
\hline Antithamnion cherminieri & 48 & $44-50$ & - & - & $\mathrm{x}$ \\
\hline "Antithamnionella atlantica & 52 & $50-53$ & - & $\mathrm{x}$ & $\mathrm{X}$ \\
\hline -Antithamnionella breviramosa & 42 & $26-87$ & - & $\mathrm{x}$ & $\mathrm{x}$ \\
\hline •Antithamnionella flagellata & 60 & $50-71$ & - & $\mathrm{x}$ & $\mathrm{X}$ \\
\hline${ }^{+}$Antithamnionella ?latiaxis & 51 & 51 & - & - & $\mathrm{x}$ \\
\hline Antithamnionella sp. & 40 & $33-58$ & - & - & - \\
\hline Apoglossum ruscifolium & 63 & $38-98$ & - & $\mathrm{x}$ & $\mathrm{X}$ \\
\hline Asparagopsis taxiformis & 34 & 34 & - & - & $\mathrm{x}$ \\
\hline •Audouinella daviesii & 44 & 44 & $\mathrm{x}$ & $\mathrm{X}$ & $\mathrm{X}$ \\
\hline 'Balliella pseudocorticata & 44 & 44 & - & - & $\mathrm{x}$ \\
\hline Botryocladia occidentalis & 36 & $25-58$ & - & $\mathrm{x}$ & $\mathrm{X}$ \\
\hline Botryocladia pyriformis & 44 & $27-59$ & - & $\mathrm{x}$ & $\mathrm{x}$ \\
\hline Branchioglossum minutum & 38 & $32-43$ & - & $\mathrm{x}$ & - \\
\hline Branchioglossum prostratum & 44 & $32-92$ & - & $\mathrm{X}$ & - \\
\hline Bryothamnion seaforthii & 28 & $28-29$ & - & $\mathrm{X}$ & $\mathrm{X}$ \\
\hline "Callithamniella tingitana & 38 & $29-42$ & - & $\mathrm{X}$ & $\mathrm{X}$ \\
\hline Callithamnion cordatum & 38 & $25-74$ & - & $\mathrm{x}$ & $\mathrm{x}$ \\
\hline
\end{tabular}


Table 5 (Continued)

\begin{tabular}{|c|c|c|c|c|c|}
\hline \multirow[t]{2}{*}{ Species } & \multicolumn{2}{|c|}{ Depth $(\mathrm{m})$} & \multicolumn{3}{|c|}{ Biogeographical affinities } \\
\hline & Mean & Range & $\begin{array}{c}\text { Cold } \\
\text { temperate }\end{array}$ & $\begin{array}{c}\text { Warm } \\
\text { temperate }\end{array}$ & Tropical \\
\hline Callithamnion halliae & 49 & $34-64$ & - & $\mathrm{x}$ & $\mathrm{x}$ \\
\hline -Callithamnion urguayense & 46 & $39-53$ & - & - & $\mathrm{x}$ \\
\hline Callophyllis sp. & 51 & $38-70$ & - & - & - \\
\hline - Calonitophyllum medium & 46 & $38-58$ & - & $\mathrm{x}$ & - \\
\hline Centroceras clavulatum & 30 & 30 & - & $\mathrm{x}$ & $\mathrm{X}$ \\
\hline${ }^{*}$ Ceramium comptum & 45 & $25-59$ & - & - & $\mathrm{X}$ \\
\hline $\begin{array}{l}\text { Ceramium fastigiatum } \mathbf{f} \text {. } \\
\text { flaccida }\end{array}$ & 32 & 32 & - & - & $\mathrm{X}$ \\
\hline Ceramium flaccidum & 30 & $26-33$ & - & $\mathrm{X}$ & $\mathrm{X}$ \\
\hline Ceramium leptozonum & 45 & $26-92$ & - & - & $\mathrm{x}$ \\
\hline Ceramium sp. & 34 & 34 & - & - & - \\
\hline Champia parvula & 38 & $24-59$ & $\mathrm{x}$ & $\mathrm{X}$ & $\mathrm{X}$ \\
\hline Chondria cnicophylla & 32 & $29-36$ & - & - & $\mathrm{X}$ \\
\hline Chondria collinsiana & 24 & 24 & - & - & $\mathrm{X}$ \\
\hline Chondria curvilineata & 34 & 34 & - & $\mathrm{x}$ & $\mathrm{x}$ \\
\hline Chondria polyrhiza & 37 & 37 & - & $\mathrm{x}$ & $\mathrm{X}$ \\
\hline Chrysymenia agardhï & 45 & $33-58$ & - & $\mathrm{x}$ & $\mathrm{x}$ \\
\hline Chrysymenia enteromorpha & 36 & $25-51$ & - & $\mathrm{x}$ & $\mathrm{x}$ \\
\hline Chrysymenia halymenioides & 41 & 41 & - & - & $\mathrm{X}$ \\
\hline Chrysymenia planifrons & 52 & $25-72$ & - & - & $\mathrm{x}$ \\
\hline - Cirrulicarpus carolinensis & 63 & $43-89$ & - & $\mathrm{x}$ & - \\
\hline Coelarthrum albertisii & 34 & $26-41$ & - & - & $\mathrm{x}$ \\
\hline "Compsothamnion thuyoides & 60 & $37-93$ & - & $\mathrm{x}$ & $\mathrm{X}$ \\
\hline Cryptonemia crenulata & 44 & $33-93$ & - & $\mathrm{x}$ & $\mathrm{x}$ \\
\hline Cryptonemia luxurians & 43 & $27-59$ & - & $\mathrm{x}$ & $x$ \\
\hline Cryptonemia sp. & 46 & $44-49$ & - & - & - \\
\hline * Cryptopleura ramosa & 49 & $38-65$ & - & - & $\mathrm{x}$ \\
\hline Dasya baillouviana & 38 & $33-44$ & $\mathrm{x}$ & $\mathrm{x}$ & $\mathrm{x}$ \\
\hline Dasya collinsiana & 29 & $25-33$ & - & - & $\mathrm{x}$ \\
\hline Dasya corymbifera & 41 & $24-58$ & - & - & $\mathrm{x}$ \\
\hline 'Dasya punicea & 49 & $33-71$ & - & - & $\mathrm{x}$ \\
\hline Dasya crouaniana & 48 & $29-72$ & - & - & $\mathrm{x}$ \\
\hline Dasya rigidula & 45 & $34-55$ & - & $\mathrm{x}$ & $\mathrm{x}$ \\
\hline Dictyurus occidentalis & 29 & $24-39$ & - & - & $\mathrm{x}$ \\
\hline "Diplothamnion jolyi & 36 & $26-42$ & - & - & $\mathrm{x}$ \\
\hline - Dudresnaya bermudensis & 45 & 45 & - & - & $\mathrm{x}$ \\
\hline Dudresnaya crassa & 53 & $43-58$ & - & $\mathrm{x}$ & $\mathrm{x}$ \\
\hline Dudresnaya patula & 46 & $33-58$ & - & - & - \\
\hline Erythrotrichia carnea & 50 & 50 & $\mathrm{x}$ & $\mathrm{x}$ & $\mathrm{x}$ \\
\hline Eucheuma isiforme & 32 & 32 & - & $\mathrm{x}$ & $\mathrm{x}$ \\
\hline Galaxaura marginata & 31 & $25-37$ & - & - & $\mathrm{x}$ \\
\hline Galaxaura oblongata & 39 & 39 & - & - & $\mathrm{x}$ \\
\hline Galaxaura obtusata & 30 & $25-37$ & - & $\mathrm{X}$ & $\mathrm{x}$ \\
\hline - Gelidiopsis planicaulis & 29 & 29 & - & - & $\mathrm{x}$ \\
\hline Gelidium americanum & 31 & $26-34$ & - & $\mathrm{x}$ & $\mathrm{x}$ \\
\hline Gloioderma atlanticum & 37 & $30-55$ & - & $\mathrm{x}$ & - \\
\hline Gloioderma blomquistii & 45 & $36-59$ & - & $\mathrm{x}$ & - \\
\hline Gloioderma rubisporum & 34 & $26-45$ & - & $\mathrm{x}$ & - \\
\hline
\end{tabular}


Table 5 (Continued)

\begin{tabular}{|c|c|c|c|c|c|}
\hline \multirow[t]{2}{*}{ Species } & \multicolumn{2}{|c|}{ Depth $(\mathrm{m})$} & \multicolumn{3}{|c|}{ Biogeographical affinities } \\
\hline & Mean & Range & $\begin{array}{c}\text { Cold } \\
\text { temperate }\end{array}$ & $\begin{array}{c}\text { Warm } \\
\text { temperate }\end{array}$ & Tropical \\
\hline Gracilaria ?cuneata & 42 & $25-58$ & - & - & $\mathrm{x}$ \\
\hline Gracilaria curtissiae & 39 & 39 & - & $\mathrm{X}$ & $\mathrm{x}$ \\
\hline Gracilaria mammillaris & 42 & $24-74$ & - & $\mathrm{x}$ & $\mathrm{x}$ \\
\hline "Graciliara occidentalis & 47 & $29-93$ & - & $\mathrm{x}$ & $\mathrm{X}$ \\
\hline "Grallatoria reptans & 54 & $53-55$ & - & - & $\mathrm{x}$ \\
\hline Griffithsia globulifera & 34 & 34 & $\mathrm{X}$ & $\mathrm{x}$ & $\mathrm{x}$ \\
\hline - Griffithsia heteromorpha & 38 & $33-41$ & - & - & $\mathrm{X}$ \\
\hline Griffithsia sp. & 44 & $28-59$ & - & - & - \\
\hline $\begin{array}{l}\text {-Grinnellia americana var. } \\
\text { caribaea }\end{array}$ & 50 & $40-74$ & - & $\mathrm{x}$ & $\mathrm{x}$ \\
\hline Gymnothamnion elegans & 72 & $58-92$ & - & - & $x$ \\
\hline Halichrysis peltata & 44 & $32-55$ & - & $\mathrm{x}$ & $\mathrm{x}$ \\
\hline Halymenia agardhii & 39 & $26-70$ & - & $\mathrm{x}$ & $\mathrm{x}$ \\
\hline Halymenia bermudensis & 39 & $24-72$ & - & $\mathrm{x}$ & $\mathrm{x}$ \\
\hline Halymenia floresia & 26 & $24-28$ & - & $\mathrm{x}$ & $x$ \\
\hline Halymenia floridana & 54 & $37-70$ & - & $\mathrm{x}$ & $\mathrm{x}$ \\
\hline Halymenia gelinaria & 53 & $27-72$ & - & $\mathrm{x}$ & $\mathrm{x}$ \\
\hline -Halymenia hancockii & 70 & $34-93$ & - & $\mathrm{x}$ & $\mathrm{x}$ \\
\hline -Halymenia integra & 44 & $28-54$ & - & - & $\mathrm{x}$ \\
\hline -Halymenia rosacea & 52 & $27-88$ & - & - & $\mathrm{x}$ \\
\hline Halymenia vinacea & 49 & $24-74$ & - & $\mathrm{x}$ & $\mathrm{x}$ \\
\hline Herposiphonia secunda & 34 & 34 & - & $\mathrm{x}$ & $\mathrm{x}$ \\
\hline $\begin{array}{l}\text { Herposiphonia secunda f. } \\
\text { tenella }\end{array}$ & 27 & $26-27$ & - & $\mathrm{x}$ & $\mathrm{x}$ \\
\hline $\begin{array}{l}\text { Heterosiphonia crispella } \\
\text { var. laxa }\end{array}$ & 36 & $25-58$ & - & $\mathrm{x}$ & $\mathrm{x}$ \\
\hline Hypnea cervicornis & 30 & $24-34$ & - & $\mathrm{x}$ & $\mathrm{x}$ \\
\hline Hypnea spinella & 30 & $25-34$ & - & - & $\mathrm{x}$ \\
\hline Hypnea volubilis & 35 & $24-50$ & - & $\mathrm{x}$ & - \\
\hline Hypoglossum anomalum & 39 & $27-70$ & - & - & $\mathrm{x}$ \\
\hline Hypoglossum hypoglossoides & 42 & 42 & - & - & $\mathrm{x}$ \\
\hline Hypoglossum tenuifolium & 42 & $24-90$ & - & $\mathrm{x}$ & $\mathrm{x}$ \\
\hline Jania adhaerens & 30 & 30 & - & $\mathrm{x}$ & $\mathrm{x}$ \\
\hline Kallymenia sp. & 54 & $34-87$ & - & - & - \\
\hline Kallymenia westii & 38 & $24-72$ & - & $\mathrm{x}$ & $\mathrm{x}$ \\
\hline Laurencia chondrioides & 32 & $26-40$ & - & - & $\mathrm{x}$ \\
\hline "Laurencia pinnatifida & 32 & $24-37$ & - & $\mathrm{x}$ & $\mathrm{x}$ \\
\hline Leptofauchea rhodymeniodes & 49 & $27-93$ & - & $\mathrm{x}$ & $\mathrm{x}$ \\
\hline Lomentaria baileyana & 42 & $25-71$ & $\mathrm{x}$ & $\mathrm{x}$ & $\mathrm{x}$ \\
\hline Lophocladia trichoclados & 49 & $33-88$ & - & $\mathrm{x}$ & $\mathrm{x}$ \\
\hline Maripelta atlantica & 68 & $37-92$ & - & - & - \\
\hline Martensia pavonia & 32 & $25-42$ & - & - & $\mathrm{x}$ \\
\hline Meristotheca floridana & 49 & $33-65$ & - & $\mathrm{x}$ & $x$ \\
\hline Micropeuce mucronata & 32 & 32 & - & $\mathrm{X}$ & $\mathrm{x}$ \\
\hline Nemastoma gelatinosum & 41 & $32-57$ & - & - & $\mathrm{x}$ \\
\hline Nitophyllum punctatum & 52 & $27-92$ & - & - & $\mathrm{x}$ \\
\hline "Nitophyllum wilkinsoniae & 41 & $27-58$ & - & $\mathrm{x}$ & - \\
\hline Peyssonnelia inamoena & 54 & $27-98$ & - & $\mathrm{x}$ & $\mathrm{x}$ \\
\hline
\end{tabular}


Table 5 (Continued)

\begin{tabular}{|c|c|c|c|c|c|}
\hline \multirow[t]{2}{*}{ Species } & \multicolumn{2}{|c|}{ Depth (m) } & \multicolumn{3}{|c|}{ Biogeographical affinities } \\
\hline & Mean & Range & $\begin{array}{l}\text { Cold } \\
\text { temperate }\end{array}$ & $\begin{array}{l}\text { Warm } \\
\text { temperate }\end{array}$ & Tropical \\
\hline "Platoma cyclocolpa & 40 & $29-48$ & - & - & $\mathrm{X}$ \\
\hline Polysiphonia binneyi & 50 & 50 & - & - & $\mathrm{X}$ \\
\hline Polysiphonia ferulacea & 41 & $39-42$ & - & $\mathrm{X}$ & $\mathrm{X}$ \\
\hline Polysiphonia havanensis & 34 & 34 & - & $\mathrm{X}$ & $\mathrm{x}$ \\
\hline Polysiphonia sphaerocarpa & 42 & $38-45$ & $\mathrm{X}$ & $\mathrm{X}$ & $\mathrm{x}$ \\
\hline -Predaea feldmanii & 40 & $26-56$ & - & $\mathrm{x}$ & $\mathrm{x}$ \\
\hline -Predaea masonii & 52 & $34-59$ & - & $\mathrm{X}$ & $\mathrm{X}$ \\
\hline •Pterocladia capillacea & 29 & 29 & - & - & $\mathrm{X}$ \\
\hline Pterothamnion plumula & 70 & $42-93$ & $\mathrm{x}$ & - & $\mathrm{x}$ \\
\hline "Rhodymenia divaricata & 42 & $27-50$ & - & $\mathrm{x}$ & - \\
\hline Rhodymenia pseudopalmata & 45 & $34-89$ & $\mathrm{x}$ & $\mathrm{X}$ & $\mathrm{X}$ \\
\hline Sarcodiotheca divaricata & 41 & $24-53$ & - & $\mathrm{x}$ & - \\
\hline Scinaia complanata & 39 & $25-58$ & - & $\mathrm{X}$ & $\mathrm{X}$ \\
\hline $\begin{array}{l}\text { Scinaia complanata var. } \\
\text { intermedia }\end{array}$ & 38 & $33-44$ & - & - & $\mathrm{X}$ \\
\hline Scinaia incrassata & 41 & $26-55$ & - & - & - \\
\hline Searlesia subtropica & 56 & $37-98$ & - & $\mathrm{X}$ & - \\
\hline Solieria filiformis & 28 & $24-34$ & $\mathrm{X}$ & $\mathrm{X}$ & $\mathrm{x}$ \\
\hline Spermothamnion investiens & 42 & $39-46$ & - & $\mathrm{x}$ & $\mathrm{x}$ \\
\hline - Spermothamnion macromeres & 41 & 41 & - & - & $\mathrm{X}$ \\
\hline Spermothamnion repens & 94 & 94 & $\mathrm{x}$ & $\mathrm{x}$ & $\mathrm{X}$ \\
\hline Spermothamnion speluncarum & 57 & $34-93$ & - & - & $\mathrm{X}$ \\
\hline Tiffaniella gorgonea & 56 & $42-90$ & - & - & $\mathrm{x}$ \\
\hline Tiffaniella saccorhiza & 60 & $43-90$ & - & - & $\mathrm{X}$ \\
\hline Titanophora incrustans & 40 & $29-50$ & - & - & $\mathrm{X}$ \\
\hline "Waldoia antillana & 38 & $25-56$ & - & - & $\mathrm{x}$ \\
\hline Wrangelia argus & 37 & $26-48$ & - & - & $\mathrm{X}$ \\
\hline Wrangelia bicuspidata & 32 & $28-37$ & - & - & $\mathrm{x}$ \\
\hline Wrightiella blodgettii & 27 & 27 & - & - & $\mathrm{x}$ \\
\hline Wrightiella tumanowiczii & 36 & $32-39$ & - & $\mathrm{X}$ & $\mathrm{X}$ \\
\hline Wurdemannia miniata & 35 & $26-58$ & - & $\mathrm{x}$ & $\mathrm{X}$ \\
\hline
\end{tabular}

1960). While these depths encompass the full depth distribution of macroalgae in many temperate areas, it was clear that macroalgae in the tropics and subtropics could grow at depths significantly greater than those that could be observed with SCUBA. Recent studies (e.g. Littler et al. 1985, 1986) have indicated that benthic macroalgae are capable of growing at much greater depths than previously believed and that the contribution to primary productivity by macroalgae growing on deep reefs in certain parts of the world could be significant.

This study has documented the species composition of a macroalgal community, at depths greater than those ever examined in such detail throughout the annual cycle. It 
Table 6. Summary of biogeographical affinities for the taxa identified during this study based on the data presented in Table $5 . \mathrm{CT}=$ Cold Temperate; $\mathrm{WT}=$ Warm Temperate; $\mathrm{T}=$ Tropical $_{\mathrm{i}}$ combinations of these indicate distribution in more than one biogeographical zone of the western North Atlantic. Taxa without a species epithet are listed as "not assignable"; taxa that are restricted to Florida near the transitional area between warm temperate and tropical zones are listed as "transitional"

\begin{tabular}{|lcccc|}
\hline $\begin{array}{c}\text { Biogeographical } \\
\text { affinity }\end{array}$ & $\begin{array}{c}\text { Chlorophyta } \\
\mathbf{n}\end{array}$ & $\begin{array}{c}\text { Phaeophyta } \\
\mathrm{n}\end{array}$ & $\begin{array}{c}\text { Rhodophyta } \\
\mathbf{n}\end{array}$ & $\begin{array}{c}\text { Total } \\
\mathrm{n}\end{array}$ \\
\hline CT & 0 & 0 & 0 & 0 \\
CT-WT & 2 & 0 & 0 & 2 \\
WT & 1 & 0 & 13 & 14 \\
WT-T & 12 & 7 & 58 & 77 \\
T & 22 & 9 & 55 & 86 \\
CT-WT-T & 4 & 1 & 11 & 16 \\
CT-T & 1 & 0 & 1 & 2 \\
Transitional & 0 & 2 & 3 & 5 \\
Not assignable & 0 & 0 & 6 & 6 \\
Total & 42 & 19 & 147 & 208 \\
\hline
\end{tabular}

has also demonstrated the high diversity that can occur in deep-water macroalgal communities in subtropical and tropical waters where high water clarity permits a much deeper euphotic zone than traditionally considered (Hanisak \& Blair, in press). Moreover, over $20 \%$ of the taxa in this study were new records for Florida; this demonstrates the importance of studying deep-water communities in order to obtain adequate floristic information for a particular area. Prior to the initiation of this submersible study, the deep-water algae of eastern Florida were known only from drift material (Eiseman, 1979).

The deep-water community of the east Florida continental shelf is characterized by species of a tropical affinity, with many species capable of growing in the warm temperate waters of the Carolinas. The community appears to consist of a relatively stable assemblage of perennial species, with an influx of incidental, shorter-lived species, particularly in the summer months. Most of the taxa present grow in much shallower waters in Florida and more tropical areas to the south; there are also a number of species that appear, based on this and previous studies, to be more characteristic of deep water. These include: the chlorophytes Cystodictyon pavonicum, Microdictyon boergesenii, ?Palmophyllum sp., Struvea pulcherrima, and S. ramosa; the phaeophytes Halopteris filicina, Sporochnus bolleanus, S. pedunculatus, and Syringoderma floridana; the rhodophytes Apoglossum ruscifolium, Chrysymenia enteromorpha, C. halymenioides, C. planifrons, Compsothamnion thuyoides, Halymenia floridana, $\mathrm{H}$. hancockii, $\mathrm{H}$. integra, $\mathrm{H}$. vinacea, $H$. rosacea, Kallymenia westii, Leptofauchea rhodymeniodes, Maripelta atlantica, Meristotheca floridana, and Searlesia subtropica. The increase in number of taxa at $31-40 \mathrm{~m}$ is probably more related to available substrate than depth per se; many species found only at depths greater than $30 \mathrm{~m}$ in this study have been found previously to grow in much shallower water.

Many basic questions about the nature of deep-water macroalgal communities are unresolved. For example, previous studies have provided conflicting evidence as to the 


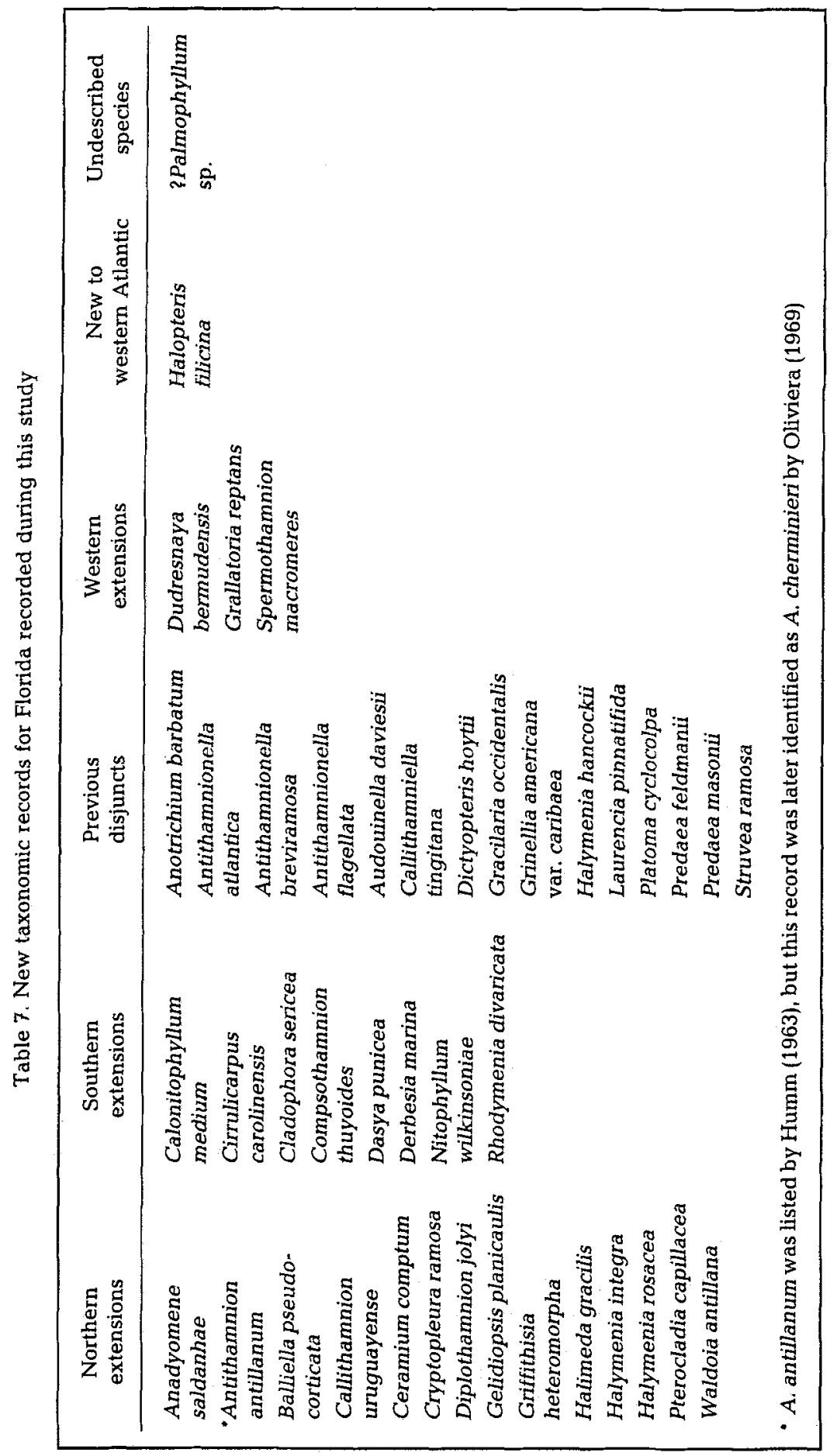


degree of seasonality in deep-water communities. Sears \& Cooper (1978) suggested that such communities in the northwest Atlantic have a high degree of seasonal stability. In the Gulf of Mexico, investigators using dredging (e.g. Dawes \& van Breedveld, 1969) had reported a similar stability in deep-water macroalgal communities. However, Cheney \& Dyer (1974) using SCUBA in 25-60 $\mathrm{m}$ in the Gulf of Mexico, described a macroalgal community with a distinct tropical affinity and a seasonal pattern of abundance and diversity, both of which were maximal in the summer. This pattern of seasonality is similar to that observed on the east Florida continental shelf in the current study as well as that observed for the offshore flora of North Carolina (Schneider, 1976; Searles, $1984 \mathrm{~b})$. We believe that differences in the apparent seasonality of deep-water communities are primarily due to methods of collections. Dredging techniques appear to preferentially harvest larger plants, which are more likely to be considered perennials, than the more direct techniques involving SCUBA and submersibles.

The zonation patterns of macroalgal divisions observed for this community are consistent with what the model of Dring (1981) predicted for clear oceanic water, i.e. rhodophytes growing deeper than chlorophytes and phaeophytes, a pattern that appears to occur throughout the world (e.g. Gilmartin, 1960; Larkum et al., 1967; Drew, 1969; Agegian \& Abbott, 1985; Hillis-Colinvaux, 1985; Littler et al., 1985, 1986). Humm \& Taylor (1961) postulated a floral diversity gradient with depth in the Gulf of Mexico; this hypothesis was supported by the submersible observations of Eiseman \& Blair (1982) who found a much greater tropical affinity of the deep-water flora of the northwest Gulf as compared to shallow-water habitats in this region. The same pattern has been observed for the North Carolina flora (Searles \& Schneider, 1980). This flora-depth relationship also appears to be the case for the east coast of Florida although a thorough analysis of shallow-water macroalgal communities for this region is lacking.

Studies of deep-water communities, such as that off eastern Florida, have important biogeographical implications. Twenty percent of the taxa identified in this study were new records to Florida; this demonstrates the necessity of extensively sampling such communities before the floristic information of a given area can be considered adequate enough for detailed biogeographical analyses. This point has been previously made by Schneider \& Searles (references cited previously) in their detailed analyses of the North Carolina offshore flora.

Perhaps most dramatic in terms of biogeographical distribution were the 15 new records for Florida that previously appeared to be distributional disjuncts. Schneider $(1976,1984)$ listed 22 taxa present in the Carolina flora with distributional disjuncts to the south; 13 of these are reported in the current study of the east coast of Florida. We believe, as Schneider (1976) suggested, that most if not all, of the remaining disjuncts are artifacts of collection. Moreover, our study substantiates a high degree of similarity between the Florida and Carolina floras; both are dominated by species with centres of distribution in the Caribbean (Schneider, 1976). The information to date suggests that the deep-water flora may be relatively continuous over a large portion of the western Atlantic. It would be desirable to conduct additional intensive floristic investigations in this region to verify this conclusion. Our observations also question the traditional practice (Stephenson \& Stephenson, 1952; Humm, 1969; Searles, 1984b) of considering Cape Canaveral as a biogeographical boundary; its utility now appears to be limited to the shallow-water flora (i. e. $<10 \mathrm{~m}$ ). 
While biogeographical investigations usually focus on latitudinal differences, there may also be significant floristic differences over short distances along a particular latitude. For example, recent studies in the Bahamas (Hanisak \& Blair, in press), including sites only $100 \mathrm{~km}$ east of the West Palm Beach stations reported herein, have demonstrated a greater predominance of green algae in the deep-water flora than fleshy red and brown algae, in contrast to the predominance of red algae off eastern Florida. The deep-water chlorophytes of the Bahamas include the species reported herein for Florida, as well as additional species of Halimeda, and Johnson-sea-linkia profunda, an apparent endemic to the Bahamas. In both Florida and the Bahamas, however, the deepest macroalgal species are crustose corallines (not considered in this manuscript). Another difference between the Florida and Bahamian deep-water macroalgal communities is the rather sharp, vertical zonation patterns that are visually and statistically dramatic in the Bahamas (Littler et al., 1985, 1986; Hanisak \& Blair, in press). These contrasts (dominance of chlorophytes versus rhodophytes, sharpness of zonation) are probably due to differences in habitat (gradual slopes versus vertical walls) and stability of conditions (water temperature and clarity are higher and more stable in the Bahamas). Lastly, the Gulf Stream between the Bahamas and Florida may serve as a distributional barrier for some deep-water macroalgae.

Clearly, deep-water macroalgal communities merit additional study in order to understand biogeographic patterns more fully. The major restraint to the study of deepwater macroalgae is the high operating costs involved with using submersibles (Earle [1985] cites a range of $\$ 7000$ to $\$ 25000$ per day, including the cost of the support vessel). Perhaps the development of simpler, less expensive one-person submersibles (Earle, 1985) or unmanned, remotely operated vehicles (ROVs) will accelerate the exploration of deep-water benthic communities by phycologists.

As access to deep-water macroalgae increases, knowledge of all aspects of the biology of these organisms will be enhanced. Continued systematic studies will lead to the description of new species and will further our knowledge of marine biogeography. Deep-water species may be useful in experimental approaches to biogeography (such as studies by Cambridge et al., 1984, 1987; Guiry et al., 1987; Lüning, 1984; McLachlan \& Bird, 1984; Yarish et al., 1984). An integration of the descriptive and experimental approaches will result in a more thorough understanding of the biogeographical patterns of marine macroalgae.

Acknowledgements. We thank N. Eiseman for initiating the program of submersible collections analysed in this paper, numerous crew members of the Johnson-Sea-Link submersibles and surface surface support ships for their invaluable assistance in making the collections, and D. Keller for assistance in preparation of the manuscript. This is Contribution No. 645 from Harbor Branch Oceanographic Institution.

\section{LITERATURE CITED}

Adey, W. H. \& MacIntyre, I. G., 1973. Crustose coralline algae: a reevalution in the geological sciences. - Bull. geol. Soc. Am. 84, 883-904.

Agegian, C. R. \& Abbott, I. A., 1985. Deep water macroalgal communities: a comparison between Penguin Bank (Hawaii) and Johnston Atoll. - Proc. int. Coral Reef Congr. 5, 47-50.

Avent, R. M. \& Stanton, F. G., 1979. Observations from research submersibles of megafaunal 
distribution on the continental margin off central eastern Florida. - Tech. Rep., Harbor Branch Found., Fort Pierce, Fla 25, 1-40.

Benz, M. C., Eiseman, N. J. \& Gallaher, E. E., 1979. Seasonal occurrence and variation in standing crop of a drift algal community in the Indian River, Florida. - Botanica mar. 22, 413-420.

Blair, S. M. \& Hall, M. O., 1981. Ten new records of deep water algae from Georgia and South Carolina. - Northeast Gulf Sci. 4, 127-129.

Cambridge, M. L., Breeman, A. M., Oosterwijk, R. van \& Hoek, C. van den., 1984. Temperature responses of some North Atlantic Cladophora species (Chlorophyceae) in relation to their geographic distribution. - Helgoländer Meeresunters. 38, 349-363.

Cambridge, M. L., Breeman, A. M., Kraak, S. \& Hoek, C. van den, 1987. Temperature responses of tropical to warm temperate Cladophora species in relation to their distribution in the North Atlantic Ocean. - Helgoländer Meeresunters. 41, 329-354.

Cheney, D. P. \& Dyer, J. P., 1974. Deep water benthic algae of the Florida Middle Ground. - Mar. Biol. 27, 185-190.

David, T. W. E., Halligan, G. H. \& Finckh, A. E., 1904. Report on dredging at Funafuti, Section VII. In: The atoll of Funafuti. Report of the Coral Reef Committee, Royal Society, 151-158.

Dawes, C. J. \& van Breedveld, J. F., 1969. Benthic marine algae. - Mem. Hourglass Cruises 1(2), $1-47$.

Doty, M. S., Gilbert, W. J. \& Abbott, I. A., 1974. Hawaiian marine algae from seaward of the algal ridge. - Phycologia 13, 345-357.

Drew, E. A., 1969. Photosynthesis and growth of attached marine algae down to 130 metres in the Mediterranean. - Proc. int. Seaweed Symp. 6r 151-159.

Dring, M. J., 1981. Chromatic adaptation of photosynthesis in benthic marine algae: an examination of its ecological significance using a theoretical model. - Limnol. Oceanogr. 26, 271-284.

Earle, S. A., 1985. Equipment for conducting research in deep waters. In: Handbook of phycological methods: ecological field methods: macroalgae. Ed. by M. M. Littler \& D. S. Littler. Cambridge Univ. Press, New York, 233-249.

Eiseman, N. J., 1978. Observations on the marine algae occurring from 30-100 meter depths on the east coast of Florida - J. Phycol. 14 (S), 25.

Eiseman, N. J., 1979. Marine algae of the east Florida continental shelf I. Some new records of Rhodophyta, including Scinaia incrassata sp. nov. (Nemalialés: Chaetangiaceae). - Phycologia $18,355-361$.

Eiseman, N. J. \& Moe, R. L., 1981. Maripelta atlantica sp. nov. (Rhodophyta, Rhodymeniales), a new deep-water alga from Florida. - J. Phycol. 17, 299-308.

Eiseman, N. J. \& Norris, J., 1981. Dudresnaya patula sp. nov., an unusual deep-water alga from Florida. - J. Phycol. 17, 186-191.

Eiseman, N. J. \& Blair, S. M., 1982. New records and range extensions of deep-water algae from East Flower Garden Bank, Northwestern Gulf of Mexico. - Contr. mar. Sci. 25, 21-26.

Gilmartin, M., 1960. The ecological distribution of the deep water algae of Enewetok Atoll. - Ecology $41,210-221$.

Guiry, M. D., Tripodi, G. \& Lüning, K., 1987. Biosystematics, genetics and upper temperature tolerance of Gigartina teedii (Rhodophyta) from the Atlantic and Mediterranean. - Helgoländer Meeresunters. 41, 283-295.

Hall, M. O. \& Eiseman, N. J., 1981. The seagrass epiphytes of the Indian River, Florida. I. Species list with descriptions and seasonal occurrence. - Botanica mar. 24, 139-146.

Hanisak, M. D. \& Blair, S. M., 1988. Deep-water benthic macroalgal communities, with emphasis on Florida and the Bahamas. In: Biogeochemical cycling and fluxes between the deep euphotic zone and other oceanic realms. Ed. by C. R. Agegian. NOAA, Washington, D. C., 3 (2) (in press).

Hillis-Colinvaux, L., 1985. Halimeda and other deep fore-reef algae at Enewetak Atoll. - Proc. int. Coral Reef Congr. 5, 9-14.

Hoek, C. van den, 1975. Phytogeographic provinces along the coasts of the northern Atlantic Ocean. - Phycologia 14, 317-330.

Hoek, C. van den, 1984. World-wide latitudinal and longitudinal seaweed distribution patterns and their possible causes, as illustrated by the distribution of Rhodophytan genera. - Helgoländer Meeresunters. 38, 227-257. 
Howe, M. A. \& Taylor, W. R., 1931. Notes on new or little known marine algae from Brazil. - Brittonia 1, 7-33.

Humm, H. J., 1956. Rediscovery of Anadyomene menziesii, a deep-water green alga from the Gulf of Mexico. - Bull. mar. Sci. Gulf Caribb. 6, 346-348.

Humm, H. J., 1963. Some new records and range extensions of Florida marine algae. - Bull. mar. Sci. Gulf Caribb. 13, 516-526.

Humm, H. J., 1969. Distribution of marine algae along the Atlantic coast of North America. Phycologia 7, 43-53.

Humm, H. J. \& Taylor, S. E., 1961. Marine Chlorophyta of the upper west coast of Florida. - Bull. mar. Sci. Gulf Carib. 11, 321-380.

Juett, L., Miller, C. J., Moore, S. J. \& Ford, E. S., 1976. Summer marine algae at Vero Beach, Florida. Fla Sci. 39, 76-80.

Kapraun, D. F., 1980. An illustrated guide to the benthic marine algae of coastal North Carolina I. Rhodophyta. Univ. North Carolina Press, Chapel Hill, 206 pp.

Kapraun, D. F., 1984. An illustrated guide to the benthic marine algae of coastal North Carolina II. Chlorophyta and Phaeophyta. Cramer, Vaduz, $173 \mathrm{pp}$.

Larkum, A. W. D., Drew, E. A. \& Crosett, R. N., 1967. The vertical distribution of attached marine algae in Malta. - J. Ecol. 55, 361-371.

Littler, M. M., Littler, D. S., Blair, S. \& Norris, J. N., 1985. Deepest known plant life discovered on an uncharted seamount. - Science, N. Y. 227, 57-59.

Littler, M. M., Littler, D. S., Blair, S. M. \& Norris, J. N., 1986. Deep-water plant communities from an uncharted seamount off San Salvador Island, Bahamas: distribution, abundance, and primary productivity. - Deep Sea Res. 33, 881-892.

Lüning, K., 1984. Temperature tolerance and biogeography of seaweeds: the marine algal flora of Helgoland (North Sea) as an example. - Helgoländer Meeresunters. 38, 305-317.

McLachlan, J. \& Bird, C. J., 1984. Geographical and experimental assessment of the distribution of Gracilaria species (Rhodophyta: Gigartinales) in relation to temperature. - Helgoländer Meeresunters. 38, 319-334.

Oliveira, F., E. C de., 1969. Algas marinhas do sul do estado do Espirito Santa (Brasil). I. Ceramiales. - Bolm Fac. Filos. Ciênc. Univ. S. Paulo (Ser. Bot.) 26 (343), 1-277.

Phillips, R. C., 1961. Seasonal aspect of the marine algal flora of St. Lucie Inlet and adjacent Indian River, Florida. - Q. Jl Fla Acad. Sci. 24, 135-147.

Schneider, C. W., 1974. North Carolina marine algae. III. A community of Ceramiales on a glass sponge from 60 meters. - Bull. mar. Sci. 24, 1093-1101.

Schneider, C. W., 1975a. North Carolina marine algae. V. Additions to the flora of Onslow Bay, including the reassignment of Fauchea peltata Taylor to Weberella Schmitz. - Br. Phycol. J. 10, 129-138.

Schneider, C. W., 1975b. North Carolina marine algae. VI. Some Ceramiales (Rhodophyta), including a new species of Dipterosiphonia. - J. Phycol. 11, 391-396.

Schneider, C. W., 1976. Spatial and temporal distributions of benthic marine algae on the continental shelf of the Carolinas. - Bull. mar. Sci. 26, 133-151.

Schneider, C. W., 1984. Studies on Antithamnionella, Callithamniella and Calloseris (Rhodophyta, Ceramiales) from North Carolina, USA. - Phycologia 23, 455-464.

Schneider, C. W. \& Eiseman, N. J., 1979. Searlesia a new genus from the western Atlantic based on Membranoptera subtropica (Rhodophyta, Delesseriaceae). - Phycologia 18, 319-324.

Schneider, C. W. \& Reading, R. P., 1987. A revision of the genus Peyssonnelia (Rhodophyta, Cryptonemiales) from North Carolina, including $P$. atlantica sp. nov. - Bull. mar. Sci. 40 (in press).

Schneider, C. W. \& Searles, R. B., 1973. North Carolina marine algae. II. New records and observations of the benthic offshore flora. - Phycologia 12, 201-211.

Schneider, C. W. \& Searles, R. B., 1975. North Carolina marine algae. IV. Further contributions from the continental shelf, including two new species of Rhodophyta. - Nova Hedwigia. 26, 83-96.

Schneider, C. W. \& Searles, R. B., 1976. North Carolina marine algae. VII. New species of Hypnea and Petroglossum (Rhodophyta, Gigartinales) and additional records of other Rhodophyta. Phycologia 15, 51-60.

Searles, R. B., 1972. North Carolina marine algae. I. Three new species from the continental shelf. Phycologia 11, 19-24. 
Searles, R. B., 1984a. North Carolina marine algae. XII. Gloioderma rubrisporum sp. nov. (Rhodophyta, Rhodymeniales). - Bull. Torrey bot. Club 111, 217-221.

Searles, R. B., 1984b. Seaweed biogeography of the mid-Atlantic coast of the United States. Helgoländer Meeresunters. 38, 259-271.

Searles, R. B. \& Schneider, C. W., 1978. A checklist and bibliography of North Carolina seaweeds. Botanica mar. 21, 99-108.

Searles, R. B. \& Schneider, C. W., 1980. Biogeographic affinities of the shallow and deepwater benthic marine algae of North Carolina. - Bull. mar. Sci. 30, 732-736.

Sears, J. R. \& Cooper, R. A., 1978. Descriptive ecology of offshore, deepwater, benthic algae in the temperate western North Atlantic Ocean. - Mar. Biol. 44, 309-314.

South, G. R. \& Tittley, I., 1986. A checklist and distributional index of the benthic marine algae of the North Atlantic Ocean. British Museum (Natural History), London, $76 \mathrm{pp}$.

Stephenson, T. A. \& Stephenson, A., 1952. Life between the tide-marks in North America. II. Northern Florida and the Carolinas. - J. Ecol. 40, 1-49.

Taylor, W. R., 1928. The marine algae of Florida with special reference to the Dry Tortugas. - Publs Carnegie Instn 379, 1-219.

Taylor, W. R., 1930. Algae collected by the Hassler, Albatross and Schmitt expeditions. I. Marine algae from Brazil. - Am. J. Bot. 17,627-634.

Taylor, W. R., 1942, Caribbean marine algae of the Allan Hancock Expedition, 1939. - Allan Hancock Atl. Exped. 2, 1-193.

Taylor, W. R., 1960. Marine algae of the eastern tropical and subtropical coasts of the Americas. Univ. Michigan Press, Ann Arbor, $870 \mathrm{pp}$.

Wiseman, D. R. \& Schneider, C. W., 1977. Investigations of the marine algae of South Carolina. I. New records of Rhodophyta. - Rhodora 78, 516-524.

Wreede, R. de \& Jones, E. C., 1973. New records of Sargassum hawailensis (Sargassaceae, Phaeophyta): a deep water species. - Phycologia 12, 59-62.

Wynne, M. J., 1986. A checklist of benthic marine algae of the tropical and subtropical western Atlantic. - Can. J. Bot. 64, 2239-2281.

Yarish, C., Breeman, A. M. \& Hoek, C. van den, 1984. Temperature, light, and photoperiod responses of some Northeast American and West European endemic rhodophytes in relation to their geographic distribution. - Helgoländer Meeresunters. 38́, 273-304. 